INTER NATIONAL MONETARY FUND
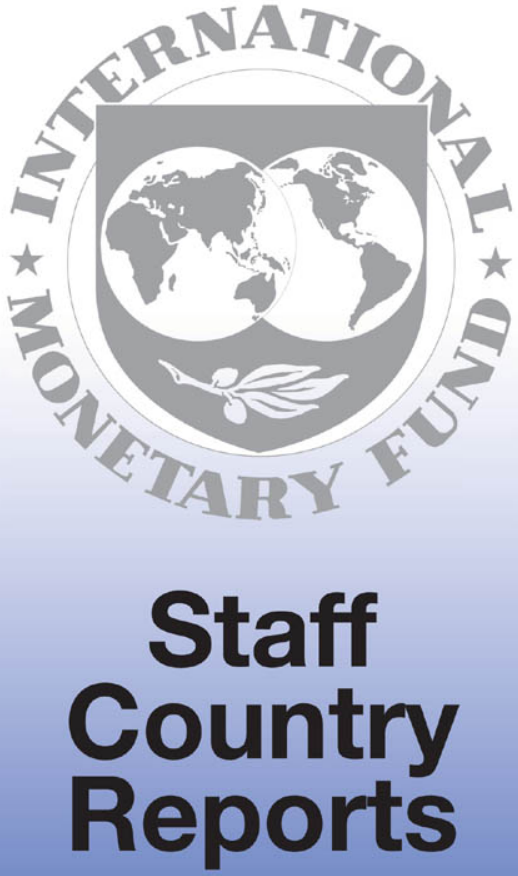


\section{Sudan: Statistical Annex}

This Statistical Annex report on Sudan was prepared by a staff team of the International Monetary Fund as background documentation for the periodic consultation with this member country. As such, the views expressed in this document are those of the staff team and do not necessarily reflect the views of the Government of Sudan or the Executive Board of the IMF.

Copies of this report are available to the public from

International Monetary Fund • Publication Services

700 19th Street, N.W. • Washington, D.C. 20431

Telephone: (202) 623-7430 - Telefax: (202) 623-7201

Telex (RCA): 248331 IMF UR

Internet: publications@imf.org

Price: $\$ 15.00$ a copy

\section{International Monetary Fund \\ Washington, D.C.}


This page intentionally left blank

(C) International Monetary Fund. Not for Redistribution 


\section{INTERNATIONAL MONETARY FUND}

\section{SUDAN}

\section{Statistical Annex}

Prepared by a staff mission consisting of Messrs. V. Sundararajan, A. Mansur, G. Shabsigh, V. Treichel, K. Thugge, and J. Ha

Approved by the Middle Eastern Department

February 18, 1998

Contents

$\underline{\text { Tables }}$

1. Origin of Gross Domestic Product, $1993 / 94-97 \ldots \ldots \ldots \ldots \ldots \ldots$

2. Indicators of Selected Agricultural Crops, $1993 / 94-1996 / 97 \ldots \ldots \ldots \ldots 4$

3. Consumer Price Index, 1992-December $1997 \ldots \ldots \ldots \ldots \ldots \ldots$

4. Central Government Operations, $1993 / 94-97 \ldots \ldots \ldots \ldots \ldots \ldots \ldots \ldots 6$

5. Monetary Survey and Factors Affecting Liquidity, 1993/94-97

6. Summary Balance of Payments, $1993 / 94-97 \ldots \ldots \ldots \ldots \ldots \ldots$

7. Main Commodities Subject to Excise, Sales, and Consumption Tax, and Their Rates, 1997

8. Selected Commodities Subject to Import Tariffs And Consumption Tax, 1997

9. Cotton Production, 1993/94-1996/97 11

10. Harvested Area, Production, and Yield of Cotton, Average 1994/95-1996/97

11. Cropped Area, Output, and Yield of Major Noncotton Crops, 1993/94-1996/97 . . . . . . . . . . . . . . . . . . . 15

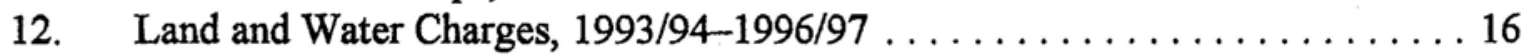

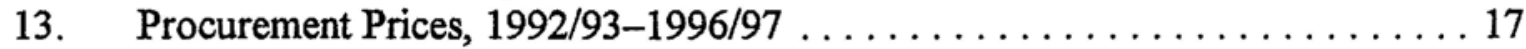

14. Cropping Patterns in the Gezira Scheme, $1993 / 94-1996 / 97 \ldots \ldots \ldots \ldots \ldots 18$

15. Manufacturing Production, $1993 / 94-97 \ldots \ldots \ldots \ldots \ldots \ldots \ldots$

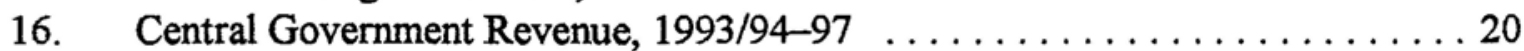

17. Central Government Expenditure, $1993 / 94-97 \ldots \ldots \ldots \ldots \ldots \ldots \ldots 21$

18. Monetary Authorities' Accounts, $1993-$ November $1997 \ldots \ldots \ldots 22$

19. Consolidated Balance Sheet of the Commercial and

Specialized Banks, 1993/94-97 
20. Commercial and Specialized Banks: Balance Sheets Assets And Deposits at Bank of Sudan, September 1997 . . . . . . . . . . . . . . 24

21. Claims of the Banking System on the Central

Government, 1993/94-97 . . . . . . . . . . . . . . . . . . . . . 25

22. Commercial Banks' Rates of Return and

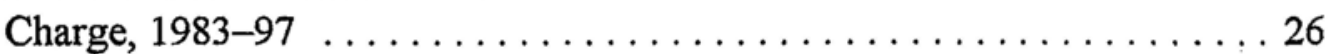

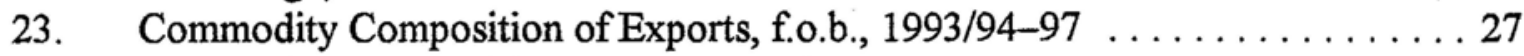

24. Commodity Composition of Imports, c.i.f., $1993 / 94-97 \ldots \ldots \ldots \ldots 28$

25. Summary Index of Export and Import Prices And Volumes, $1993 / 94-97$. . . . . . . . . . . . . . . . . . . . . . 29

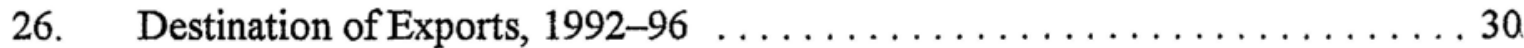

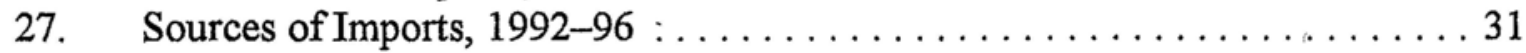

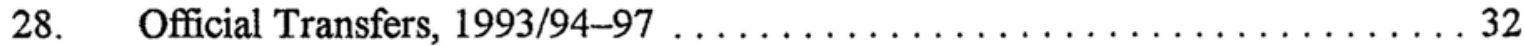

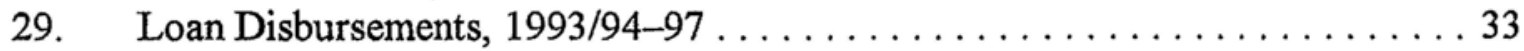

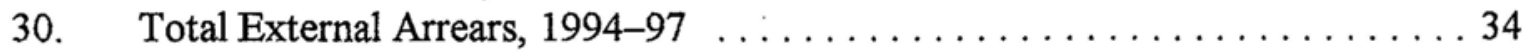

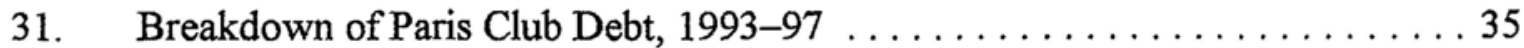

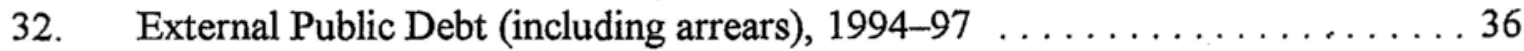

33. External Public Debt Service Obligations, 1993/94-97 . . . . . . . . . . 37

34. Imports of Petroleum Products, and Landed Costs,

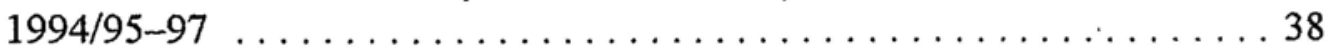

35. Average Capacity Utilization in the Industrial Sector, 1993/94-97

\section{$\underline{\text { Appendix }}$}

I. The Tax System of Sudan 
Table 1. Sudan: Origin of Gross Domestic Product, 1993/94-97 1/

\begin{tabular}{|c|c|c|c|c|c|c|}
\hline & 1993/94 & $1994 / 95$ & $1995 / 96$ & 1995 & 1996 & 1997 \\
\hline & \multicolumn{6}{|c|}{ (In billions of Sudanese pounds) } \\
\hline GDP at constant factor cost & 7,812 & 8,165 & 8,518 & 9,967 & 10,438 & 11,016 \\
\hline Agriculture & 2,793 & 3,178 & 3,471 & 4,177 & 4,696 & 5,288 \\
\hline Crops & 1,528 & 1,835 & 2,051 & 1,953 & 2,245 & 2,699 \\
\hline Irrigated crops & 991 & 1,102 & 1,392 & 1,025 & 1,392 & 1,572 \\
\hline Rainfed mechan. crops & 206 & 273 & 228 & 266 & 229 & 361 \\
\hline Rainfed tradit. crops & 330 & 460 & 431 & 663 & 624 & 766 \\
\hline Livestock & 990 & 1,010 & 1,060 & 1,892 & 2,091 & 2,215 \\
\hline Forestry and fishing & 275 & 333 & 360 & 331 & 360 & 374 \\
\hline Industry & 1,383 & 1,407 & 1,498 & 1,406 & 1,509 & 1,542 \\
\hline Mining and quarrying & 12 & 13 & 90 & 13 & 90 & 105 \\
\hline Manufacturing & 715 & 653 & 671 & 652 & 682 & 690 \\
\hline Electricity and water & 191 & 193 & 199 & 193 & 199 & 207 \\
\hline Construction & 465 & 548 & 538 & 548 & 538 & 540 \\
\hline Services & 3,637 & 3,580 & 3,549 & 4,385 & 4,233 & 4,186 \\
\hline Government services & 704 & 630 & 585 & 837 & 780 & 719 \\
\hline Other services & 2,933 & 2,950 & 2,965 & 3,548 & 3,453 & 3,467 \\
\hline GDP deflator $(1981 / 82=100)$ & 20,702 & 35,772 & 84,779 & 41,928 & 97,884 & 143,836 \\
\hline GDP at current factor cost & $1,617,240$ & $2,920,781$ & $7,221,722$ & $4,179,000$ & $10,217,132$ & $15,845,036$ \\
\hline Indirect taxes less subsidies & 63,157 & 130,823 & 140,000 & 135 & 274 & 274 \\
\hline \multirow[t]{2}{*}{ GDP at current market prices } & $1,680,397$ & $3,051,604$ & $7,361,722$ & $4,179,135$ & $10,217,408$ & $15,845,310$ \\
\hline & \multicolumn{6}{|c|}{ (Annual percentage change) } \\
\hline GDP at constant factor cost & 4.3 & 4.5 & 4.3 & $\therefore$ & 4.7 & 5.5 \\
\hline Agriculture & 6.2 & 13.8 & 9.2 & $\ldots$ & 12.4 & 12.6 \\
\hline Crops & 1.2 & 20.1 & 11.8 & $\cdots$ & 14.9 & 20.2 \\
\hline Irrigated crops & 18.0 & 11.2 & 26.3 & $\ldots$ & 35.9 & 12.9 \\
\hline Rainfed mechan. crops & -47.0 & 32.5 & -16.5 & $\ldots$ & -13.8 & 57.6 \\
\hline Rainfed tradit. crops & 18.0 & 39.2 & -6.3 & $\cdots$ & -5.9 & 22.8 \\
\hline Livestock & 11.2 & 2.0 & 5.0 & $\ldots$ & 10.5 & 5.9 \\
\hline Forestry and fisheries & 19.6 & 21.1 & 8.1 & $\ldots$ & 8.8 & 3.9 \\
\hline Industry & 4.1 & 1.8 & 6.4 & $\cdots$ & 7.3 & 2.2 \\
\hline Mining and quarrying & 0.0 & 8.3 & 592.3 & $\ldots$ & 581.8 & 16.7 \\
\hline Manufacturing & -2.1 & -8.7 & 2.7 & $\cdots$ & 4.6 & 1.2 \\
\hline Electricity and water & 9.1 & 1.2 & 3.0 & $\cdots$ & 3.2 & 4.0 \\
\hline Construction & 12.9 & 17.8 & -1.8 & $\ldots$ & -1.8 & 0.4 \\
\hline Services & 3.0 & -1.6 & -0.9 & $\ldots$ & -3.5 & -1.1 \\
\hline Government services & 7.3 & -10.5 & -7.2 & $\cdots$ & -6.8 & -7.8 \\
\hline \multirow[t]{2}{*}{ Other services } & 2.0 & 0.6 & 0.5 & $\cdots$ & -2.7 & 0.4 \\
\hline & \multicolumn{6}{|c|}{ (Sources of GDP growth, in percentage points) } \\
\hline GDP at constant factor cost & 4.3 & 4.5 & 4.3 & $\ldots$ & 4.7 & 5.5 \\
\hline Agriculture & 2.2 & 4.9 & 3.6 & $\cdots$ & 5.1 & 5.7 \\
\hline Crops & 0.2 & 3.9 & 2.6 & $\ldots$ & 3.6 & 4.3 \\
\hline Irrigated crops & 2.0 & 1.4 & 3.6 & $\ldots$ & 5.9 & 1.7 \\
\hline Rainfed mechan. crops & -2.4 & 0.9 & -0.6 & $\ldots$ & -0.4 & 1.3 \\
\hline Rainfed tradit. crops & 0.7 & 1.7 & -0.4 & $\ldots$ & -0.3 & 1.4 \\
\hline Livestock & 1.3 & 0.3 & 0.6 & $\ldots$ & 1.3 & 1.2 \\
\hline Forestry and fisheries & 0.6 & 0.7 & 0.3 & $\ldots$ & 0.4 & 0.1 \\
\hline Industry & 0.7 & 0.3 & 1.1 & $\ldots$ & 1.3 & 0.3 \\
\hline Mining and quarrying & 0.0 & 0.0 & 0.9 & $\ldots$ & 6.1 & 0.1 \\
\hline Manufacturing & -0.2 & -0.8 & 0.2 & $\ldots$ & 0.4 & 0.1 \\
\hline Electricity and water & 0.2 & 0.0 & 0.1 & $\ldots$ & 0.1 & 0.1 \\
\hline Construction & 0.7 & 1.1 & -0.1 & $\ldots$ & -0.1 & 0.0 \\
\hline Services & 1.4 & -0.7 & -0.4 & $\ldots$ & -1.4 & -0.5 \\
\hline Government services & 0.6 & -0.9 & -0.6 & $\ldots$ & -0.5 & -0.6 \\
\hline Other services & 0.8 & 0.2 & 0.2 & $\ldots$ & -0.9 & 0.1 \\
\hline
\end{tabular}

Sources: Ministry of Economics, Planning, and Investment; and Fund staff estimates.

1/ Data were reported based on fiscal year until 1995, and on calendar year from 1995. 
Table 2. Sudan: Indicators of Selected Agricultural Crops, 1993/94-1996/97 1/

(Area in thousands of feddans; production in thousands of metric tons; and yield in kilograms per feddan)

\begin{tabular}{|c|c|c|c|c|c|c|c|c|c|c|c|c|}
\hline & \multirow{2}{*}{\multicolumn{3}{|c|}{ Irrigated }} & \multicolumn{6}{|c|}{ Rainfed } & \multirow{2}{*}{\multicolumn{3}{|c|}{ Total }} \\
\hline & & & & \multicolumn{3}{|c|}{ Mechanized } & \multicolumn{3}{|c|}{ Traditional } & & & \\
\hline & Area & Production & Yield & Area & Production & Yield & Area & Production & Yield & Area & Production & Yield \\
\hline \multicolumn{13}{|l|}{ Cotton } \\
\hline $1993 / 94$ & 278 & 144 & 518 & 14 & 4 & 260 & 0 & 0 & 0 & 292 & 148 & 507 \\
\hline $1994 / 95$ & 420 & 256 & 610 & 18 & 3 & 167 & 0 & 0 & 0 & 438 & 259 & 591 \\
\hline 1995 & 534 & 300 & 562 & 55 & 7 & 177 & 0 & 0 & 0 & 589 & 307 & 521 \\
\hline 1996 Est. & 586 & 286 & 488 & 83 & 7 & 84 & 21 & 4 & 190 & 690 & 297 & 430 \\
\hline 1997 Est. & 591 & 309 & 523 & 0 & 0 & 0 & 103 & 313 & 223 & 594 & 622 & 1,187 \\
\hline \multicolumn{13}{|c|}{ Dura (Sorghum) } \\
\hline $1993 / 94$ & 904 & 602 & 666 & 7,896 & 1,473 & 187 & 2,352 & 311 & 132 & 11,152 & 2,386 & 214 \\
\hline $1994 / 95$ & 1,181 & 726 & 615 & 7,730 & 1,623 & 210 & 4,392 & 878 & 200 & 13,303 & 3,227 & 243 \\
\hline 1995 & 737 & 512 & 695 & 6,843 & 1,379 & 202 & 3,697 & 542 & 147 & 11,277 & 2,433 & 216 \\
\hline 1996 Est. & 877 & 888 & 1,013 & 10,345 & 2,388 & 231 & 4,380 & 903 & 202 & 15,602 & 4,179 & 268 \\
\hline 1997 Est. & 875 & 894 & 1,021 & 10,324 & 2,402 & 233 & 4,387 & 885 & 202 & 15,586 & 4,181 & 268 \\
\hline \multicolumn{13}{|l|}{ Groundnuts } \\
\hline $1993 / 94$ & 293 & 254 & 867 & 0 & 0 & 0 & 1,565 & 174 & 111 & 1,858 & 428 & 230 \\
\hline $1994 / 95$ & 299 & 262 & 876 & 0 & 0 & 0 & 1,814 & 452 & 249 & 2,113 & 714 & 338 \\
\hline 1995 & 350 & 326 & 931 & 0 & 0 & 0 & 2,230 & 412 & 185 & 2,580 & 738 & 286 \\
\hline 1996 Est. & 359 & 342 & 953 & 0 & 0 & 0 & 1,892 & 473 & 249 & 2,251 & 815 & 362 \\
\hline 1997 Est. & $\cdots$ & $\cdots$ & $\cdots$ & 0 & 0 & 0 & $\cdots$ & $\cdots$ & $\cdots$ & $\cdots$ & $\cdots$ & $\cdots$ \\
\hline \multicolumn{13}{|l|}{ Wheat } \\
\hline $1993 / 94$ & 851 & 475 & 558 & 0 & 0 & 0 & 0 & 0 & 0 & 851 & 475 & 558 \\
\hline $1994 / 95$ & 656 & 443 & 675 & 0 & 0 & 0 & 6 & 2 & 300 & 662 & 445 & 672 \\
\hline 1995 & 691 & 520 & 753 & 0 & 0 & 0 & 18 & 7 & 400 & 709 & 527 & 743 \\
\hline 1996 Est. & 757 & 628 & 830 & 0 & 0 & 0 & 27 & 14 & 519 & 784 & 642 & 819 \\
\hline 1997 Est. & 782 & 636 & 813 & 0 & 0 & 0 & 0 & 0 & 0 & 782 & 636 & 813 \\
\hline \multicolumn{13}{|l|}{ Millet } \\
\hline $1993 / 94$ & 2 & 1 & 300 & 162 & 27 & 166 & 2,381 & 193 & 81 & 2,545 & 221 & 87 \\
\hline $1994 / 95$ & 10 & 2 & 180 & 76 & 10 & 132 & 7,621 & 961 & 126 & 7,707 & 973 & 126 \\
\hline 1995 & 8 & 2 & 250 & 58 & 8 & 138 & 5,692 & 375 & 66 & 5,758 & 385 & 67 \\
\hline 1996 Est. & 10 & 2 & 200 & 168 & 27 & 161 & 3,711 & 411 & 111 & 3,889 & 440 & 113 \\
\hline 1997 Est. & 898 & 643 & 982 & 0 & 0 & 0 & 3,593 & 384 & 107 & 4,491 & 1,027 & 229 \\
\hline \multicolumn{13}{|l|}{ Total $1 /$} \\
\hline $1993 / 94$ & 2,328 & 1,476 & 634 & 8,072 & 1,504 & 186 & 6,298 & 678 & 108 & 16,698 & 3,658 & 219 \\
\hline $1994 / 95$ & 2,566 & 1,689 & 658 & 7,824 & 1,636 & 269 & 13,833 & 2,293 & 166 & 24,223 & 5,618 & 232 \\
\hline 1995 & 2,320 & 1,660 & 716 & 6,956 & 1,394 & 200 & 11,637 & 1,336 & 115 & 20,913 & 4,390 & 210 \\
\hline 1996 Est. & 2,589 & 2,146 & 829 & 10,596 & 2,422 & 229 & 10,031 & 1,805 & 180 & 23,216 & 6,373 & 275 \\
\hline 1997 Est. & 3,505 & 2,824 & 806 & 10,324 & 2,402 & 233 & 9,975 & 2,055 & 206 & 23,704 & 7,281 & 307 \\
\hline
\end{tabular}

Source: Ministry of Agriculture.

1/ Excludes fruits and vegetables that account for a large portion of agricultural output.

CInternational Monetary Fund. Not for Redistribution 
Table 3. Sudan: Consumer Price Index, 1992-December 1997 1/

(Index, January 1990 = 100)

\begin{tabular}{|c|c|c|c|}
\hline & Index & $\begin{array}{r}\text { 12-Month } \\
\text { Percentage } \\
\text { Change }\end{array}$ & $\begin{array}{r}\text { Monthly } \\
\text { Percentage } \\
\text { Change }\end{array}$ \\
\hline 1992 & 863 & 150 & $\ldots$ \\
\hline 1993 & 1,888 & 119 & $\ldots$ \\
\hline 1994 & 3,881 & 106 & $\ldots$ \\
\hline 1995 & 6,629 & 71 & $\ldots$ \\
\hline 1996 & 14,203 & 114 & $\ldots$ \\
\hline 1997 & 18,754 & 32 & \\
\hline \multicolumn{4}{|l|}{1996} \\
\hline January & 7,059 & 84 & 6.5 \\
\hline February & 7,662 & 97 & 8.5 \\
\hline March & 8,141 & 103 & 6.2 \\
\hline April & 8,768 & 116 & 7.7 \\
\hline May & 9,252 & 119 & 5.5 \\
\hline June & 11,612 & 162 & 25.5 \\
\hline July & 12,412 & 157 & 6.9 \\
\hline August & 13,686 & 165 & 10.3 \\
\hline September & 13,963 & 157 & 2.0 \\
\hline October & 14,595 & 159 & 4.5 \\
\hline November & 14,519 & 133 & -0.5 \\
\hline December & 14,203 & 114 & -2.2 \\
\hline \multicolumn{4}{|l|}{1997} \\
\hline January & 14,215 & 101 & 0.1 \\
\hline February & 14,730 & 92 & 3.6 \\
\hline March & 15,450 & 90 & 4.9 \\
\hline April & 15,330 & 75 & -0.8 \\
\hline May & 15,712 & 70 & 2.5 \\
\hline June & 16,461 & 42 & 4.8 \\
\hline July & 17,022 & 37 & 3.4 \\
\hline August & 18,083 & 32 & 6.2 \\
\hline September & 17,731 & 27 & -1.9 \\
\hline October & 17,350 & 19 & -2.2 \\
\hline November & 18,418 & 27 & 6.2 \\
\hline December & 18,754 & 32 & 1.8 \\
\hline
\end{tabular}

Sources: Statistics Department of the Ministry of Finance.

1/ Middle income index covering greater Khartoum area. 
Table 4. Sudan: Central Government Operations, 1993/94-97 1/

\begin{tabular}{|c|c|c|c|c|c|}
\hline & $1993 / 94$ & $1994 / 95$ & 1995 & 1996 & $\frac{\text { Est. }}{1997}$ \\
\hline & \multicolumn{5}{|c|}{ (In billions of Sudanese pounds) } \\
\hline Total revenue & 106 & 285 & 365 & 629 & 1,080 \\
\hline Tax revenue & 106 & 212 & 289 & 533 & 820 \\
\hline Nontax revenue & 0 & 73 & 76 & 97 & 260 \\
\hline \multicolumn{6}{|l|}{ Total expenditure (including } \\
\hline interest arrears) & 356 & 671 & 1,005 & 2,722 & 2,868 \\
\hline Expenditure excluding interest arrears & 199 & 338 & 504 & 975 & 1,207 \\
\hline Current expenditure $2 /$ & 151 & 278 & 376 & 727 & 1,114 \\
\hline Wages and salaries & 29 & 49 & 60 & 220 & 375 \\
\hline Goods and services & 65 & 98 & 156 & 244 & 458 \\
\hline Transfers & 35 & 80 & 38 & 56 & 47 \\
\hline Interest paid & 5 & 25 & 40 & 100 & 101 \\
\hline Other $2 /$ & 17 & 26 & 82 & 107 & 133 \\
\hline Extrabudgetary & 0 & 0 & 58 & 192 & 0 \\
\hline Capital expenditure and net lending & 0 & 60 & 67 & 56 & 93 \\
\hline Interest arrears on external debt & 157 & 333 & 501 & 1,747 & 1,661 \\
\hline Overall deficit (on an accrual basis) & -250 & -386 & -640 & $-2,093$ & $-1,788$ \\
\hline Overall deficit (on a cash basis) & -93 & -53 & -135 & -346 & -127 \\
\hline Financing & 93 & 53 & 131 & 346 & 127 \\
\hline External & 21 & 33 & 42 & 22 & 26 \\
\hline Domestic bank borrowing (net) $3 /$ & 79 & 23 & 89 & 324 & 101 \\
\hline Exceptional domestic borrowing & -7 & -3 & 0 & $\ldots$ & ... \\
\hline \multirow[t]{2}{*}{ Float } & $\cdots$ & $\cdots$ & 4 & $\cdots$ & $\cdots$ \\
\hline & \multicolumn{5}{|c|}{ (As percent of GDP) } \\
\hline Total revenue & 7.8 & 9.4 & 8.7 & 6.2 & 6.8 \\
\hline \multicolumn{6}{|l|}{ Total expenditure (including } \\
\hline interest arrears) & 21.2 & 22.0 & 24.0 & 26.7 & 18.2 \\
\hline Total expenditure (excluding interest arrears) & 11.9 & 11.1 & 12.0 & 9.6 & 7.6 \\
\hline \multicolumn{6}{|l|}{ Total expenditure excluding interest } \\
\hline payments and arrears & 11.6 & 10.3 & 11.1 & 8.6 & 7.0 \\
\hline Development expenditure & 1.7 & 2.0 & 1.6 & 0.5 & 0.6 \\
\hline Overall balance (on an accrual basis) & -13.4 & -12.7 & -15.3 & -20.5 & -11.3 \\
\hline Overall balance (on a cash basis) & -4.1 & -1.7 & -3.2 & -3.4 & -0.8 \\
\hline Primary balance & -3.8 & -0.9 & -2.3 & -2.4 & -0.2 \\
\hline
\end{tabular}

Sources: Ministry of Finance; Bank of Sudan; and Fund staff projections.

1/ Starting in 1996, all official accounting moved from a fiscal year (July-June) to a calendar year basis.

2/ Starting in 1995, includes expenditures on commodity aid.

3/ As reported in the monetary survey by the Bank of Sudan. 
Table 5. Sudan: Monetary Survey and Factors Affecting Liquidity, 1993/94-1997

(In billions of Sudanese pounds, unless otherwise stated)

\begin{tabular}{|c|c|c|c|c|c|}
\hline & $1993 / 94$ & $1994 / 95$ & 1995 & 1996 & $\frac{\text { Est. }}{1997}$ \\
\hline Net foreign assets & -601 & $-1,642$ & $-2,572$ & $-4,462$ & $-5,115$ \\
\hline Bank of Sudan & -684 & $-1,472$ & $-2,712$ & $-4,719$ & $-5,515$ \\
\hline Commercial banks & 83 & 100 & 141 & 257 & 401 \\
\hline Net domestic credit & 214 & 272 & 399 & 817 & 992 \\
\hline Net claims on central government & 143 & 160 & 251 & 472 & 573 \\
\hline Claims on nongovernment sector & 71 & 112 & 148 & 342 & 419 \\
\hline Other items (net) & 36 & 113 & 205 & 187 & 240 \\
\hline Counterpart to valuation changes & 669 & 1,745 & 2,674 & 4,624 & 5,480 \\
\hline Money and quasi-money & 317 & 489 & 706 & 1,166 & 1,597 \\
\hline Currency & 112 & 177 & 249 & 444 & 585 \\
\hline Demand deposits & 77 & 129 & 168 & 328 & 432 \\
\hline Savings and investment deposits in LSd & 28 & 41 & 62 & 87 & 132 \\
\hline \multirow[t]{2}{*}{ Deposits in foreign currency } & 100 & 143 & 227 & 306 & 449 \\
\hline & \multicolumn{5}{|c|}{ (Percentage change) } \\
\hline Net domestic credit & 82.5 & 27.1 & 46.7 & 104.8 & 21.4 \\
\hline Net claims on central government & 53.2 & 11.9 & 56.9 & 88.0 & 21.4 \\
\hline \multirow[t]{2}{*}{ Credit to nongovernement sectors $1 /$} & 29.3 & 57.7 & 32.1 & 131.1 & 22.5 \\
\hline & \multicolumn{5}{|c|}{ (Change as percent of initial broad money) } \\
\hline Net foreign assets & -113.8 & -328.5 & -326.3 & -267.9 & -56.0 \\
\hline Net domestic credit & 49.2 & 18.6 & 32.7 & 59.3 & 15.0 \\
\hline Net claims on central government & 31.7 & 5.5 & 22.0 & 31.7 & 8.4 \\
\hline Claims on non-government sectors $1 /$ & 17.5 & 13.1 & 10.7 & 27.5 & 6.7 \\
\hline Other items, net & 17.6 & 24.3 & 34.8 & -2.6 & 4.5 \\
\hline Valuation counterpart & 136.1 & 339.9 & 333.0 & 276.3 & 73.4 \\
\hline \multirow[t]{2}{*}{ Money and quasi-money } & 89.1 & 54.2 & 74.2 & 65.2 & 37.0 \\
\hline & \multicolumn{5}{|c|}{ (In millions of U.S. dollars) } \\
\hline \multicolumn{6}{|l|}{ Memorandum items: } \\
\hline Net foreign assets of Bank of Sudan 2/ & $-3,166$ & $-3,324$ & $-3,237$ & $-3,232$ & $-3,203$ \\
\hline Foreign currency deposits & 325 & 272 & 271 & 210 & 261 \\
\hline Velocity of money $3 /$ & 7.2 & 7.0 & 7.8 & 10.7 & 11.4 \\
\hline \multicolumn{6}{|l|}{ Deposits in foreign currency (as } \\
\hline percent of broad money) & 31.6 & 29.2 & 32.2 & 26.2 & 28.1 \\
\hline
\end{tabular}

Sources: Bank of Sudan; and Fund staff projections.

1/ Private sector and nonfinancial public enterprises.

2/ Net reserve assets.

3/ Nominal GDP divided by the monthly average of money and quasi-money during the period. 
Table 6. Sudan: Summary Balance of Payments, 1993/94-97

\begin{tabular}{|c|c|c|c|c|c|}
\hline & 1993/94 & $1994 / 95$ & 1995 & 1996 & $\frac{\text { Est. }}{1997}$ \\
\hline & \multicolumn{5}{|c|}{ (In millions of U.S. dollars) } \\
\hline Trade balance & -724.8 & -601.7 & -663.2 & -927 & -975 \\
\hline Exports, f.o.b. & 502.6 & 421.7 & 555.6 & 620 & 580 \\
\hline Cotton & 95.9 & 95.5 & 123 & 128 & 111 \\
\hline Gum arabic & 81 & 44.7 & 51.4 & 29 & 23 \\
\hline Livestock & 89.8 & 61.7 & 84.1 & 81 & 73 \\
\hline Sesame & 76.7 & 60.6 & 80.6 & 141 & 117 \\
\hline Other & 159.2 & 159.2 & 216.6 & 241 & 255 \\
\hline Imports, c.i.f. & $-1,227.4$ & $-1,023.4$ & $-1,218.8$ & $-1,547$ & $-1,555$ \\
\hline Foodstuffs & -204.8 & -181.4 & -214.5 & -232 & -243 \\
\hline Petroleum & -230.4 & -178.2 & -194.2 & -306 & -299 \\
\hline Manufactured goods & -293.2 & -228.6 & -276.6 & -298 & -285 \\
\hline Other & -499.1 & -435.2 & -533.5 & -658 & -593 \\
\hline Oil-pipeline related exports & 0 & 0 & 0 & -53 & -135 \\
\hline Services (net) & -10 & -5.1 & -5.5 & -29 & -25 \\
\hline Receipts & 113.7 & 74.7 & 131.6 & 52 & 33 \\
\hline Payments & -123.7 & -79.8 & -137 & -81 & -59 \\
\hline Income (net) & -885.7 & -936.3 & -914.4 & -953 & $-1,099$ \\
\hline Receipts & 1.4 & 1.3 & 1.9 & 5 & 14 \\
\hline Payments & -887.1 & -937.6 & -916.3 & -958 & $-1,113$ \\
\hline \multicolumn{6}{|l|}{ Of which: } \\
\hline Public interest payments due $1 /$ & -868.3 & -931.2 & -912.6 & -956 & $-1,108$ \\
\hline (Total cash payments) & -20.4 & -30.4 & -26.9 & -18.6 & -4.2 \\
\hline Current transfers (net) & 123.7 & 122.1 & 103.9 & 61 & 107 \\
\hline Private transfers (net) & 54.4 & 81.4 & 60.1 & 21 & 92 \\
\hline Public transfers (Cash and commodity aid) & 69.3 & 40.7 & 43.8 & 40 & 15 \\
\hline Current account balance & $-1,496.8$ & $-1,421$ & $-1,479.2$ & $-1,848$ & $-1,992$ \\
\hline Excluding public transfers & $-1,566.1$ & $-1,461.7$ & $-1,523$ & $-1,888$ & $-2,007$ \\
\hline Capital account & 18.8 & 11.6 & 10.6 & 8 & 4 \\
\hline Project aid & 18.8 & 11.6 & 10.6 & 8 & 4 \\
\hline Financial Account (net) & -324.5 & 114.2 & 75.0 & -123 & -118 \\
\hline Disbursements & 97.7 & 76.7 & 86.0 & 27 & 17 \\
\hline Cash and commodity loans & 45.2 & 20.9 & 22.9 & 12.7 & 0.2 \\
\hline Project loans & 52.5 & 55.8 & 63.1 & 15.9 & 16.3 \\
\hline Amortization & -374 & -311.8 & -298.0 & -280 & -233 \\
\hline Short-term capital flows (net) $2 /$ & 9.9 & 273.3 & 330.6 & 121 & 40 \\
\hline Commercial banks reserve (net) (increase -) & -58 & 76 & -54 & 7 & 58 \\
\hline Errors \& omissions and other private capital & 603.5 & 114.6 & 282.0 & 785 & 826 \\
\hline Overall balance & $-1,199.0$ & $-1,180.7$ & $-1,110.5$ & $-1,178$ & $-1,280$ \\
\hline
\end{tabular}


Table 6. Sudan: Summary Balance of Payments, 1993/94-97

\begin{tabular}{|c|c|c|c|c|c|}
\hline & $1993 / 94$ & $1994 / 95$ & 1995 & 1996 & $\begin{array}{r}\text { Est. } \\
1997\end{array}$ \\
\hline Change in official reserves (increase -) & 32.5 & -1.3 & -22.5 & -13 & -23 \\
\hline IMF (net) & 49.5 & -2.3 & -2.5 & -10 & -23 \\
\hline Change in arrears on repurchases & 0 & -32.4 & -39.7 & -36 & -57 \\
\hline Change in arrears on charges $3 /$ & 49.5 & 30.1 & 37.2 & 26 & 34 \\
\hline Other & -17 & 1 & -20.0 & -3 & 1 \\
\hline Debt relief and restructuring 4/ & 0 & 29 & 29 & 0 & 0 \\
\hline \multirow[t]{2}{*}{ Net change in non-Fund arrears } & $1,166.5$ & 1,153 & 1,104 & 1,191 & 1,302 \\
\hline & \multicolumn{5}{|c|}{ (In percent of GDP) } \\
\hline Trade balance & -11.5 & -8.5 & -9.3 & -12.2 & -12.0 \\
\hline Current account & -23.8 & -20.1 & -20.8 & -24.4 & -24.4 \\
\hline Non-interest Current Account & -10 & -6.9 & -8 & -11.8 & -10.8 \\
\hline Overall balance & -19.1 & -16.7 & -15.7 & -15.5 & -15.7 \\
\hline Exports, f.o.b. & 8 & 6 & 7.8 & 8.2 & 7.1 \\
\hline Imports, c.i.f. & 19.5 & 14.4 & 17.2 & 20.4 & 19.1 \\
\hline External debt service due & 19.8 & 17.5 & 16.9 & 16.3 & 16.4 \\
\hline \multicolumn{6}{|l|}{ Total external debt service paid } \\
\hline (including arrears) & 0.5 & 0.9 & 1.0 & 0.7 & 0.8 \\
\hline \multicolumn{6}{|l|}{ Of which: } \\
\hline Interest payments & 0.3 & 0.4 & 0.4 & 0.2 & 0.1 \\
\hline \multicolumn{6}{|l|}{ External outstanding debt (in billions } \\
\hline of U.S. dollars) & 17.8 & 18.7 & 19.8 & 20.7 & 21.7 \\
\hline (In percent of GDP) & 283.2 & 263.9 & 279 & 272.5 & 266.3 \\
\hline (In percent of current account receipts) & $2,276.8$ & $2,509.9$ & $1,912.3$ & $2,303.6$ & $2,162.3$ \\
\hline External arrears (in billions of U.S. dollars) & 13.6 & 14.8 & 16.3 & 17.4 & 18.7 \\
\hline \multicolumn{6}{|l|}{ External debt service due (in percent } \\
\hline c.a. receipts) & 159.1 & 166.9 & 115.9 & 137.7 & 133.5 \\
\hline \multicolumn{6}{|l|}{$\begin{array}{l}\text { Actual debt payments (in percent of } \\
\text { c.a. receipts) }\end{array}$} \\
\hline (Including arrears payments to the Fund) & 3.9 & 9.2 & 7.0 & 6.3 & 6.1 \\
\hline
\end{tabular}

Source: Staff estimates based on information provided by the Sudanese authorities.

1/ Includes estimates of late interest accrued during the year and Fund special charges.1

$2 /$ Net short-term trade and other credit facilities of the Bank of Sudan and commercial banks.

$3 /$ Including refunds of burden sharing.

4/ In May 1995, reflects a restructuring debt operation with the African Development Bank Group amounting to US\$29 million. 
Table 7. Sudan: Main Commodities Subject to Excise, Sales, and Consumption Taxes, and their Rates, 1997

(In percent, unless otherwise stated)

\begin{tabular}{|c|c|c|c|}
\hline Commodity & Excise Tax & Sales Tax & $\begin{array}{c}\text { Consumption } \\
\text { Tax }\end{array}$ \\
\hline Tobacco $(\mathbf{F})$ & 150 & n.a. & 10 \\
\hline Cement (F) & LSd 100,000 per ton & n.a. & 10 \\
\hline Soft drinks (F/S) & 7 & n.a. & 10 \\
\hline Biscuits and cakes (S) & 6 & 0 & n.a. \\
\hline Tomato paste (S) & 0 & 0 & n.a. \\
\hline Flour $(\mathrm{F})$ & 0 & n.a. & 0 \\
\hline Edible oils (S) & 2 & 0 & n.a. \\
\hline Ready made clothes (S) & 0 & 0 & n.a. \\
\hline Shoes (S) & 0 & 0 & n.a. \\
\hline Sweets (S) & 2 & 0 & n.a. \\
\hline Soap-toilette (S) & 10 & $\mathbf{0}$ & n.a. \\
\hline Soap-regular (S) & $\mathbf{0}$ & 10 & n.a. \\
\hline Perfumes/cosmetics (S) & 20 & 10 & n.a. \\
\hline Toothpaste (S) & 0 & 10 & n.a. \\
\hline Tires (F) & 5 & n.a. & 10 \\
\hline Tubes and pipes (S) & 10 & 10 & n.a. \\
\hline Batteries/electric flashlights (F) & 10 & n.a. & 10 \\
\hline Household materials (S) & 10 & 10 & n.a. \\
\hline Paints and polishes (F) & 20 & n.a. & 10 \\
\hline
\end{tabular}

Source: Data provided by the Sudanese authorities.

F: Revenues collected are transfered to the Central Government.

S: Revenues collected are transfered to the State Governments.

F/S: Revenues collected are shared by the Central and State Governments. 
Table 8. Sudan: Selected Commodities Subject to Import Tariffs and Consumption Tax, 1997

(In percent; unless otherwise stated)

\begin{tabular}{|c|c|c|c|}
\hline Commodity & $\begin{array}{l}\text { Consumption } \\
\text { Tax }\end{array}$ & $\begin{array}{l}\text { Import } \\
\text { Duty }\end{array}$ & Total Rate \\
\hline Motor oil and lubricants & 10 & 5 & 15 \\
\hline Rice & 10 & 5 & 15 \\
\hline Wheat flour & 10 & 5 & 5 \\
\hline Printed boods and booklets & $\ldots$ & 5 & 5 \\
\hline Sacks and bags for packing & 2 & 5 & 7 \\
\hline Insecticides and pesticides & 2 & 5 & 7 \\
\hline Tractors & 10 & 5 & 15 \\
\hline Harvesters and parts & 10 & 5 & 15 \\
\hline Coffee & 2 & 10 & 12 \\
\hline Natural yeasts & 10 & 5 & 15 \\
\hline Tea & 2 & 5 & 7 \\
\hline Vegetable oil & 10 & 5 & 15 \\
\hline Additives for mineral oil & 10 & 5 & 15 \\
\hline Margarine & 10 & 5 & 15 \\
\hline Caustic soda & 10 & 5 & 15 \\
\hline Zinc oxide and peroxide & 10 & 5 & 15 \\
\hline Chemical products & 10 & 5 & 15 \\
\hline Polymerization products & 10 & 50 & 60 \\
\hline Synthetic rubber & 10 & 5 & 15 \\
\hline Natural resine & $\ldots$ & 5 & 5 \\
\hline Writing paper & $\ldots$ & 5 & 5 \\
\hline Paper board boxes & 10 & 10 & 20 \\
\hline Yarn of man-made fibers & 10 & 5 & 15 \\
\hline Glass bottles, etc. & 10 & 5 & 15 \\
\hline Iron and steel wire & 10 & 5 & 15 \\
\hline Metal containers & 10 & 5 & 15 \\
\hline Combustion engines & 10 & 5 & 15 \\
\hline Pumps & 10 & 5 & 15 \\
\hline Machinery, plant equipment & 10 & 5 & 15 \\
\hline Excavating machineries & 10 & 5 & 15 \\
\hline Machineries and accessories & 10 & 5 & 15 \\
\hline Auxiliary machinery & 10 & 5 & 15 \\
\hline Sewing machines & 10 & 5 & 15 \\
\hline Tapes, valves, etc. & 10 & 5 & 15 \\
\hline Compressors & 10 & 5 & 15 \\
\hline Bell bearings & 10 & 5 & 15 \\
\hline Transformers and generators & 10 & 5 & 15 \\
\hline Other electric goods & 10 & 5 & 15 \\
\hline Electric fans & 10 & 10 & 20 \\
\hline Electric ignition & 10 & 5 & 15 \\
\hline Electric lighting for cars & 10 & 30 & 40 \\
\hline Electric apparatus & 10 & 5 & 15 \\
\hline Parts for tractors & 10 & 5 & 15 \\
\hline Parts for motor vehicles & 10 & 5 & 15 \\
\hline Motocycles & 10 & 10 & 20 \\
\hline
\end{tabular}


Table 8. Sudan: Selected Commodities Subject to Import Tariffs and Consumption Tax, 1997

(In percent; unless otherwise stated)

\begin{tabular}{|c|c|c|c|}
\hline Commodity & $\begin{array}{l}\text { Consumption } \\
\text { Tax }\end{array}$ & $\begin{array}{l}\text { Import } \\
\text { Duty }\end{array}$ & Total Rate \\
\hline $\begin{array}{l}\text { Cycles and parts } \\
\text { Lead pencils } \\
\text { Trucks and lorries } \\
\text { Mini-buses } \\
\text { Buses }\end{array}$ & $\begin{array}{l}10 \\
10 \\
10 \\
10 \\
10\end{array}$ & $\begin{array}{r}5 \\
30 \\
5 \\
5 \\
5\end{array}$ & $\begin{array}{l}15 \\
40 \\
15 \\
15 \\
15\end{array}$ \\
\hline $\begin{array}{l}\text { Paper } \\
\text { Cinnamon } \\
\text { Cloves } \\
\text { Cement } \\
\text { Sliced wood } \\
\text { Cables } \\
\text { Polishing and crushing stones } \\
\text { Sheets and plates of iron } \\
\text { Iron and steel products } \\
\text { Iron and steel bars and rods } \\
\text { Tubes and pipe fittings } \\
\text { Needles } \\
\text { Razors and blades } \\
\text { Locks and padlocks } \\
\text { Lamps and lighting fittings } \\
\text { Electric parts } \\
\text { Televisions } \\
\text { Pharmaceutical glassware } \\
\text { Refined sugar } \\
\text { Other papers and paper boards } \\
\text { Tableware and other } \\
\text { Glassware } \\
\text { Nails, tacks, stapples, etc. }\end{array}$ & $\begin{array}{l}10 \\
10 \\
10 \\
10 \\
10 \\
10 \\
10 \\
10 \\
10 \\
10 \\
10 \\
10 \\
10 \\
10 \\
10 \\
10 \\
10 \\
10 \\
10 \\
10 \\
10 \\
10 \\
10\end{array}$ & $\begin{array}{r}5 \\
10 \\
10 \\
10 \\
5 \\
5 \\
30 \\
30 \\
30 \\
30 \\
10 \\
10 \\
10 \\
30 \\
51 \\
30 \\
30 \\
5 \\
30 \\
30 \\
30 \\
50 \\
5\end{array}$ & $\begin{array}{l}15 \\
20 \\
20 \\
20 \\
15 \\
15 \\
40 \\
40 \\
40 \\
40 \\
20 \\
20 \\
20 \\
40 \\
15 \\
40 \\
40 \\
15 \\
40 \\
40 \\
40 \\
60 \\
15\end{array}$ \\
\hline $\begin{array}{l}\text { Heavy duty pickups } \\
\text { Sauces and seasonings } \\
\text { Soups and broths } \\
\text { Perfumes and cosmetics } \\
\text { Rubber tires } \\
\text { Cotton fabrics } \\
\text { Other cotton fabrics } \\
\text { Air coolers } \\
\text { Primary cells and batteries } \\
\text { Cars } \\
\text { Pick-ups }\end{array}$ & $\begin{array}{l}10 \\
10 \\
10 \\
10 \\
10 \\
10 \\
10 \\
10 \\
10 \\
10 \\
10\end{array}$ & $\begin{array}{r}100 \\
30 \\
10 \\
50 \\
50 \\
75 \\
75 \\
50 \\
100 \\
100 \\
100\end{array}$ & $\begin{array}{r}110 \\
40 \\
20 \\
60 \\
60 \\
85 \\
85 \\
60 \\
110 \\
110 \\
110\end{array}$ \\
\hline
\end{tabular}

Source: Data provided by the Sudanese Authorities. 
Table 9. Sudan: Cotton Production, 1993/94-1996/97 1/

(In thousands of bales) $2 /$

\begin{tabular}{lrrrr}
\hline & $1993 / 94$ & $1994 / 95$ & $1995 / 96$ & $1996 / 97$ \\
\hline $\begin{array}{l}\text { Total production } \\
\text { Extra long-staple } \\
\quad \text { (Barakat) }\end{array}$ & 245 & 436 & 518 & 520 \\
$\begin{array}{l}\text { Long-staple } \\
\text { (Shambat "B") }\end{array}$ & 37 & 77 & 111 & 125 \\
$\begin{array}{l}\text { Medium-staple (Acala) } \\
\text { Short-staple }\end{array}$ & 41 & 31 & 0 & 3 \\
\hline
\end{tabular}

Sources: Ministry of Agriculture; and Fund staff estimates.

1/ Based on crops cycle.

2/ One bale of lint weighs 420 pounds or approximately 0.191 metric tons. 
Table 10. Sudan: Harvested Area, Production, and Yield of Cotton, Average 1994/95-1996/97

(Area in thousands of feddans; production in thousands of metric tons; and yield in kilograms per feddan)

\begin{tabular}{|c|c|c|c|c|c|c|c|c|c|}
\hline & \multirow{3}{*}{ Area } & & \multirow{3}{*}{$\begin{array}{l}\text { Average } \\
\text { Yield }\end{array}$} & & & \multirow{3}{*}{$\begin{array}{r}\text { Average } \\
\text { Yield }\end{array}$} & & & \multirow{2}{*}{$\begin{array}{r}\text { Average } \\
\text { _Yield }\end{array}$} \\
\hline & & Procuction & & Area & Production & & Area & Production & \\
\hline & & $1994 / 95$ & & \multicolumn{2}{|r|}{$1995 / 96$} & & \multicolumn{2}{|r|}{$1996 / 97$} & \\
\hline \multicolumn{10}{|l|}{ Irrigated sector } \\
\hline Egyptian & 130 & 67 & 515 & 134 & 66 & 493 & 148 & 62 & 419 \\
\hline Blue Nile & 15 & 7 & 495 & 19 & 8 & 421 & 4 & 1 & 311 \\
\hline Gezira and Manegil & 102 & 57 & 559 & 96 & 52 & 538 & 131 & 58 & 439 \\
\hline Tokar & 9 & 1 & 135 & 9 & 1 & 134 & 4 & 1 & 212 \\
\hline Suki & 0 & 0 & 0 & 0 & 0 & 0 & 0 & 0 & 0 \\
\hline White Nile & 0 & 0 & 0 & 10 & 5 & 500 & 9 & 2 & 212 \\
\hline New Halfa & 4 & 2 & 490 & 0 & 0 & 0 & 0 & 0 & 0 \\
\hline Rahad & 0 & 0 & 0 & 0 & 0 & 0 & 0 & 0 & 0 \\
\hline Acala & 290 & 189 & 652 & 400 & 234 & 585 & 438 & 224 & 511 \\
\hline Rahad & 59 & 43 & 736 & 68 & 48 & 708 & 58 & 43 & 739 \\
\hline Zeidab & 0 & 0 & 0 & 0 & 0 & 0 & 0 & 0 & 0 \\
\hline Gezira & 150 & 96 & 637 & 203 & 127 & 657 & 200 & 114 & 569 \\
\hline Blue Nile & 0 & 0 & 0 & 13 & 5 & 385 & 18 & 17 & 354 \\
\hline New Halfa & 50 & 32 & 357 & 60 & 28 & 460 & 58 & 26 & 442 \\
\hline Suki & & & & 16 & 7 & 442 & 12 & 5 & 396 \\
\hline \multicolumn{10}{|l|}{ Rainfed sector } \\
\hline American & 18 & 3 & 167 & 55 & 7 & 127 & 104 & 11 & 106 \\
\hline Blue Nile & 8 & 1 & 158 & 23 & 5 & 165 & 53 & 4 & 81 \\
\hline Gedaref & 6 & 1 & 145 & 13 & 1 & 112 & 2 & $\ldots$ & 125 \\
\hline Nuba mountains & 2 & 0 & 158 & 5 & 1 & 150 & 4 & 1 & 117 \\
\hline Abu Habil & 2 & 1 & 395 & 4 & 4 & 113 & 23 & 2 & 90 \\
\hline Equatoria & 0 & 0 & 0 & 0 & 0 & 0 & 17 & 3 & 150 \\
\hline White Nile & & & & 1 & $\ldots$ & 141 & $\begin{array}{l}4 \\
1\end{array}$ & $\begin{array}{c}1 \\
\ldots\end{array}$ & $\begin{array}{r}135 \\
90\end{array}$ \\
\hline Total & 438 & 259 & 591 & 589 & 307 & 521 & 690 & 297 & 430 \\
\hline
\end{tabular}


Table 11. Sudan: Cropped Area, Output, and Yield of Major Noncotton Crops, 1993/94-1996/97

\begin{tabular}{|c|c|c|c|c|}
\hline & $1993 / 94$ & $1994 / 95$ & $1995 / 96$ & $1996 / 97$ \\
\hline & \multicolumn{4}{|c|}{ (In thousands of feddans) } \\
\hline \multicolumn{5}{|c|}{ (2) } \\
\hline Sorghum & 11,152 & 13,303 & 11,277 & 15,602 \\
\hline Irrigated & 903 & 1,181 & 737 & 877 \\
\hline Rainfed & 10,249 & 12,122 & 10,540 & 14,725 \\
\hline Millet & 2,543 & 7,707 & 5,758 & 3,889 \\
\hline Groundnuts & 1,858 & 2,113 & 2,580 & 2,251 \\
\hline Irrigated & 293 & 299 & 350 & 359 \\
\hline Rainfed & 1,565 & 1,814 & 2,230 & 1,892 \\
\hline Sesame & 2,931 & 3,206 & 3,556 & 4,430 \\
\hline Wheat & 851 & 662 & 709 & 784 \\
\hline & \multicolumn{4}{|c|}{ (In thousands of metric tons) } \\
\hline \multicolumn{5}{|l|}{ Output } \\
\hline Sorghum & 2,386 & 3,648 & 2,433 & 4,179 \\
\hline Irrigated & 602 & 726 & 512 & 888 \\
\hline Rainfed & 1,784 & 2,501 & 1,920 & 3,291 \\
\hline Millet & 221 & 973 & 885 & 440 \\
\hline Groundnuts & 428 & 714 & 738 & 815 \\
\hline Irrigated & 254 & 262 & 326 & 342 \\
\hline Rainfed & 174 & 452 & 412 & 473 \\
\hline Sesame & 175 & 170 & 313 & 416 \\
\hline Wheat & 475 & 445 & 527 & 642 \\
\hline Gum arabic & 24 & 27 & 25 & $\ldots$ \\
\hline & \multicolumn{4}{|c|}{ (In kilograms per feddan) } \\
\hline \multicolumn{5}{|l|}{ Yields } \\
\hline Sorghum & 214 & 238 & 216 & 268 \\
\hline Irrigated & 666 & 615 & 695 & 1,013 \\
\hline Rainfed & 174 & 207 & 182 & 223 \\
\hline Millet & 87 & 126 & 67 & 113 \\
\hline Groundnuts & 231 & 338 & 286 & 362 \\
\hline Irrigated & 867 & 876 & 931 & 953 \\
\hline Rainfed & 111 & 249 & 185 & 249 \\
\hline Sesame & 60 & 53 & 88 & 94 \\
\hline Wheat & 558 & 672 & 743 & 819 \\
\hline
\end{tabular}

Sources: Ministry of Agriculture; Ministry of Economics, Planning, and Investment; and Gum Arabic Corporation. 
Table 12. Sudan: Land and Water Charges, 1993/94-1996/97

(In Sudanese pounds per feddan)

\begin{tabular}{|c|c|c|c|c|c|c|c|c|}
\hline & \multicolumn{4}{|c|}{ Gezira Scheme } & \multicolumn{4}{|c|}{ Other Schemes 1/ } \\
\hline & $1993 / 94$ & $1994 / 95$ & $1995 / 96$ & $1996 / 97$ & $1993 / 94$ & $1994 / 95$ & $1995 / 96$ & $1996 / 97$ \\
\hline Cotton & 1,650 & 2,350 & 17,281 & 25,000 & 1,800 & 2,500 & 20,710 & 30,000 \\
\hline Wheat & 1,300 & 1,800 & 12,961 & 21,000 & 1,300 & 2,200 & 15,532 & 20,500 \\
\hline Groundnuts & 1,300 & 1,300 & 12,961 & 21,000 & 1,300 & 2,200 & 15,532 & 30,500 \\
\hline Sorghum & 1,300 & 1,300 & 12,100 & 21,000 & 1,300 & 2,200 & 14,497 & 26,200 \\
\hline Vegetables & 5,000 & 3,200 & 25,150 & 45,000 & 5,000 & 4,250 & 28,993 & 42,500 \\
\hline
\end{tabular}

Source: Ministry of Agriculture.

1/ Includes Rahad. 
Table 13. Sudan: Procurement Prices, 1992/93-1996/97

(In Sudanese pounds per kantar)

\begin{tabular}{|c|c|c|c|c|c|}
\hline & $1992 / 93$ & $1993 / 94$ & $1994 / 95$ & $1995 / 96$ & $1996 / 97$ \\
\hline \multicolumn{6}{|l|}{ Cotton $1 /$} \\
\hline Extra long-staple (Barakat) & & $14,9602 /$ & $19,1252 /$ & $49,6992 /$ & $\cdots$ \\
\hline Grade 1 & 6,090 & & & & \\
\hline 2 & 5,990 & & & & \\
\hline 3 & 5,890 & & & & \\
\hline 4 & 5,790 & & & & \\
\hline 5 & 5,690 & & & & \\
\hline 6 & 5,590 & & & & \\
\hline 7 & 5,490 & & & & \\
\hline 8 & 5,390 & & & & \\
\hline 9 & 5,290 & & & & \\
\hline Long-staple (Shambat "B") & & $11,7032 /$ & $27,3402 /$ & $49,7612 /$ & $\ldots$ \\
\hline Grade 1 & 4,800 & & & & \\
\hline 2 & 4,700 & & & & \\
\hline 3 & 4,600 & & & & \\
\hline 4 & 4,500 & & & & \\
\hline 5 & 4,400 & & & & \\
\hline 6 & 4,300 & & & & \\
\hline Medium-staple (Acala) & & $13,7942 / 3 /$ & $30,0002 / 3 /$ & $38,5202 / 3 /$ & $\ldots$ \\
\hline Grade 1 & 4,550 & & & & \\
\hline 2 & 4,450 & & & & \\
\hline 3 & 4,350 & & & & \\
\hline 4 & 4,250 & & & & \\
\hline 5 & 4,150 & & & & \\
\hline Wheat (LSd per ton) & 12,000 & 38,500 & 143,000 & 220,000 & 471,470 \\
\hline \multicolumn{6}{|l|}{ Gum arabic } \\
\hline (ex El-Obeid) & 2,789 & 17,500 & 25,000 & 25,000 & 25,000 \\
\hline
\end{tabular}

Sources: Ministry of Agriculture; and Sudan Cotton Company.

1/ Prices are in Sudanese pounds per kantar of seed cotton. One kantar of seed cotton weighs 315 pounds and yields about 106 pounds of lint in the case of long-staple cotton, 95 pounds for Shambat "B", and about 116 pounds of lint in the case of medium-staple cotton. Procurement prices were abolished in 1996/97.

2/ Average prices according to formula: US\$ export (f.o.b.) price less 11.5 percent (1 percent for Sudan Cotton Co., 0.5 percent quay dues, and 10 percent for export tax) equals "ex-store" price; "ex-store" price converted at average official exchange rate less 4 percent ( 2 percent each for Sudan cotton Company and Reserve Fund) equals net procurement price for cotton.

3/ New Halfa Agricultural Scheme. 
Table 14. Sudan: Cropping Patterns in the Gezira Scheme, 1993/94-1996/97

\begin{tabular}{|c|c|c|c|c|}
\hline & $1993 / 94$ & $1994 / 95$ & $1995 / 96$ & $1996 / 97$ \\
\hline & \multicolumn{4}{|c|}{ (In thousands of feddans) } \\
\hline Total area cultivated & 1,398 & 1,293 & 1,361 & 1,419 \\
\hline Groundnuts & 178 & 191 & 231 & 242 \\
\hline Wheat & 531 & 393 & 390 & 385 \\
\hline Sorghum & 540 & 499 & 385 & 405 \\
\hline Cotton & 94 & 155 & 299 & 331 \\
\hline \multirow[t]{2}{*}{ Other noncotton } & 55 & 55 & 56 & 56 \\
\hline & \multicolumn{4}{|c|}{ (As percent of total area) } \\
\hline Total area cultivated & 100.0 & 100.0 & 100.0 & 100.0 \\
\hline Groundnuts & 12.7 & 14.8 & 17.0 & 17.1 \\
\hline Wheat & 38.0 & 30.4 & 28.7 & 27.1 \\
\hline Sorghum & 38.6 & 38.6 & 28.3 & 28.5 \\
\hline Cotton & 6.7 & 11.9 & 22.0 & 23.3 \\
\hline \multirow[t]{2}{*}{ Other noncotton } & 4.0 & 4.3 & 4.1 & 3.9 \\
\hline & \multicolumn{4}{|c|}{ (In thousands of metric tons) } \\
\hline \multicolumn{5}{|l|}{ Output } \\
\hline Groundnuts & 149 & 159 & 231 & 242 \\
\hline Wheat & 282 & 230 & 254 & 270 \\
\hline \multirow[t]{2}{*}{ Sorghum } & 394 & 338 & 271 & 465 \\
\hline & \multicolumn{4}{|c|}{ (In kilograms per feddan) } \\
\hline \multicolumn{5}{|l|}{ Yield } \\
\hline Groundnuts & 837 & 350 & 1,000 & 1,000 \\
\hline Wheat & 531 & 600 & 560 & 700 \\
\hline Sorghum & 730 & 683 & 703 & 1,149 \\
\hline
\end{tabular}

Sources: Sudan Gezira Board; and Ministry of Agriculture. 
Table 15. Sudan: Manufacturing Production, 1993/94-97

\begin{tabular}{|c|c|c|c|c|c|}
\hline & $1993 / 94$ & 1994/95 & 1995 & 1996 & $\begin{aligned} \text { Est. } \\
1997\end{aligned}$ \\
\hline \multicolumn{6}{|l|}{ Food and beverages } \\
\hline Flour (thousand tons) & 423 & 300 & 350 & 360 & 324 \\
\hline Sugar (thousand tons) & 425 & 428 & 450 & 460 & 500 \\
\hline \multicolumn{6}{|l|}{ Vegetable oils } \\
\hline (thousand tons) & 90 & 90 & 70 & 118 & 90 \\
\hline \multicolumn{6}{|l|}{ Soft drinks } \\
\hline (million dozen bottles) & 28 & 29 & 33 & 29 & 23 \\
\hline \multicolumn{6}{|l|}{ Textiles and apparel } \\
\hline Textiles (million yards) & 75 & 30 & 24 & 19 & 36 \\
\hline Shoes (million pairs) & 1.3 & 5.2 & 6.0 & 9.0 & 24.0 \\
\hline \multicolumn{6}{|l|}{ Other consumer goods } \\
\hline Cigarettes (tons) & 1,935 & 1,417 & 1,317 & 1,300 & 1,138 \\
\hline Tires (thousands) & 111 & 133 & 186 & 198 & 60 \\
\hline \multicolumn{6}{|l|}{ Intermediate goods } \\
\hline Cement (thousand tons) & 186 & 249 & 199 & 380 & 288 \\
\hline \multicolumn{6}{|l|}{ Petroleum products } \\
\hline (thousand tons) & 1,000 & $\ldots$ & $\ldots$ & $\ldots$ & 1,145 \\
\hline
\end{tabular}

Source: Ministry of Industry. 
Table 16. Sudan: Central Government Revenue, 1993/94-97 1/

\begin{tabular}{|c|c|c|c|c|c|}
\hline & $1993 / 94$ & $1994 / 95$ & 1995 & 1996 & 1997 \\
\hline & \multicolumn{5}{|c|}{ (In billions of Sudanese pounds) } \\
\hline Total Revenue & 131.9 & 285.2 & 365.4 & 629.5 & $1,073.9$ \\
\hline Tax Revenue & 106.5 & 212.3 & 288.7 & 532.6 & 814.2 \\
\hline Taxes on income and profits & 37.9 & 75.1 & 104.9 & 177.9 & 216.6 \\
\hline Personal income tax & 1.3 & 4.3 & 7.1 & 14.3 & 21.2 \\
\hline Business profit tax & 30.2 & 64.7 & 83.6 & 135.2 & 132.1 \\
\hline Sudanese working abroad & 4.2 & 5.0 & 10.2 & 21.1 & 41.1 \\
\hline Development tax & $\ldots$ & $\ldots$ & 0.3 & 2.8 & 2.9 \\
\hline Other $1 /$ & 2.2 & 1.1 & 3.7 & 45.0 & 19.3 \\
\hline Taxes on goods and services & 28.5 & 54.1 & 69.8 & 78.0 & 178.3 \\
\hline Excise duties & 17.7 & 44.1 & 59.8 & 71.3 & 178.3 \\
\hline Sales taxes & 10.8 & 10.0 & 10.0 & 6.7 & 0.0 \\
\hline \multicolumn{6}{|l|}{ Taxes on international trade } \\
\hline and transactions & 37.2 & 74.9 & 105.9 & 276.8 & 419.3 \\
\hline Import duties & 16.9 & 42.1 & 60.2 & 167.4 & 239.0 \\
\hline Export tax & 3.9 & 7.2 & 8.0 & 21.0 & 31.1 \\
\hline Consumption tax & 13.4 & 24.2 & 32.3 & 62.8 & 26.6 \\
\hline Other & 3.0 & 1.4 & 5.3 & 25.6 & 122.6 \\
\hline Other taxes $2 /$ & 2.9 & 8.2 & 8.1 & 0.0 & 0.0 \\
\hline Nontax revenue & 25.4 & 72.9 & 76.7 & 96.9 & 259.7 \\
\hline Fees and charges on public services & 2.5 & 4.6 & 45.3 & 57.1 & 64.1 \\
\hline Charges on land and water & 0.2 & 0.7 & 3.2 & $\therefore$ & $\ldots$ \\
\hline \multicolumn{6}{|l|}{ Public enterprises profits, } \\
\hline interest, rent, and dividends & 5.1 & 5.2 & 9.3 & 30.2 & 49.5 \\
\hline Commodity price differentials (oil) & . & 37.6 & $\ldots$ & $\ldots$ & 57.6 \\
\hline Commodity price differentials (sugar) & 7.5 & 4.7 & 9.3 & $\ldots$ & 14.3 \\
\hline Sales of Sudanese petroleum & . & $\ldots$ & $\ldots$ & $\ldots$ & 6.6 \\
\hline Receipts from sales of public enterprises & 0.5 & 7.8 & 2.0 & 3.0 & 0.7 \\
\hline Land sales & 0.9 & 2.6 & 2.0 & $\ldots$ & 0.0 \\
\hline Pension contributions & $\ldots$ & .. & ... & $\ldots$ & 0.0 \\
\hline Other $3 /$ & 8.6 & 9.6 & 5.4 & 5.6 & 66.9 \\
\hline \multirow{2}{*}{ Loan repayments } & 0.1 & 0.1 & 0.1 & 0.9 & 0.0 \\
\hline & \multicolumn{5}{|c|}{ (In percent of GDP) } \\
\hline Total revenue & 7.8 & 9.4 & 8.7 & 6.2 & 6.8 \\
\hline Tax revenue & 6.3 & 7.0 & 6.9 & 5.2 & 5.1 \\
\hline \multirow[t]{2}{*}{ Nontax revenue } & 1.5 & 2.4 & 1.8 & 0.9 & 1.6 \\
\hline & \multicolumn{5}{|c|}{ (In billions of Sudanese pounds) } \\
\hline Nominal GDP & 1,680 & 3,052 & 4,179 & 10,217 & 15,845 \\
\hline
\end{tabular}

Source: Ministry of Finance and Economic Planning; and Fund staff estimates.

1/ Starting in 1996, all official accounting moved from a fiscal year (July-June) to a calendar year basis.

2/ Includes rent tax and capital gains tax.

3/ Includes stamp duty, car licences, agricultural taxes, and airport departure tax. 
Table 17. Sudan: Central Government Expenditure, 1993/94-97

\begin{tabular}{|c|c|c|c|c|c|}
\hline & 1993/94 & $1994 / 95$ & 1995 & 1996 & 1997 \\
\hline & \multicolumn{5}{|c|}{ (In billions of Sudanese pounds) } \\
\hline Total expenditure & 356.4 & 671.3 & $1,002.0$ & $2,722.1$ & $2,868.0$ \\
\hline $\begin{array}{l}\text { Total expenditure (excluding } \\
\text { interest arrears) }\end{array}$ & 199.3 & 337.6 & 503.7 & 925.0 & $1,206.1$ \\
\hline $\begin{array}{l}\text { Current expenditure (excluding } \\
\text { interest arrears) } \\
\text { Wages and salaries } \\
\text { Goods and services } \\
\text { Ministries } \\
\text { Defense } 1 / \\
\text { Other } 2 / \\
\text { Current transfers } \\
\text { To regions } \\
\text { To institutions } \\
\text { Interest paid }\end{array}$ & $\begin{array}{r}151.9 \\
32.7 \\
66.7 \\
22.1 \\
41.1 \\
3.5 \\
30.9 \\
4.7 \\
26.2 \\
5.0\end{array}$ & $\begin{array}{r}278.1 \\
48.9 \\
105.9 \\
32.8 \\
65.5 \\
7.6 \\
39.1 \\
20.0 \\
19.1 \\
34.0\end{array}$ & $\begin{array}{r}376.0 \\
60.0 \\
155.7 \\
55.4 \\
80.6 \\
19.7 \\
38.5 \\
16.7 \\
21.7 \\
39.6\end{array}$ & $\begin{array}{r}727.0 \\
220.0 \\
244.0 \\
78.8 \\
95.2 \\
70.0 \\
56.0 \\
35.4 \\
20.6 \\
100.0\end{array}$ & $\begin{array}{r}1,113.0 \\
334.6 \\
329.1 \\
84.6 \\
163.0 \\
81.5 \\
87.4 \\
47.4 \\
40.0 \\
101.3\end{array}$ \\
\hline $\begin{array}{l}\text { General reserves, (including for } \\
\text { emergency, exchange rate } \\
\text { changes, and other expenditures) }\end{array}$ & 16.6 & 50.2 & 82.1 & 107.0 & 260.6 \\
\hline $\begin{array}{l}\text { Capital expenditure and net lending } \\
\text { Development expenditure } \\
\text { Foreign financed } \\
\text { Domestically financed } \\
\text { Agriculture and industry funds } \\
\text { Equity } \\
\text { Net lending }\end{array}$ & $\begin{array}{r}28.8 \\
25.3 \\
16.4 \\
7.4 \\
1.4 \\
0.8 \\
2.7\end{array}$ & $\begin{array}{r}59.5 \\
58.8 \\
31.0 \\
23.5 \\
4.3 \\
0.7 \\
\cdots\end{array}$ & $\begin{array}{r}67.0 \\
67.0 \\
39.0 \\
23.7 \\
4.3 \\
\ldots \\
\ldots\end{array}$ & $\begin{array}{r}56.0 \\
56.0 \\
34.1 \\
21.9 \\
\cdots \\
\cdots \\
\cdots\end{array}$ & $\begin{array}{r}93.1 \\
93.1 \\
26.4 \\
66.7 \\
0.0 \\
0.0 \\
0.0\end{array}$ \\
\hline Extrabudgetary outlays & 18.6 & $\ldots$ & 58.0 & 192.0 & 0.0 \\
\hline \multirow[t]{2}{*}{ Interest arrears } & 157.1 & 333.7 & 498.3 & $1,747.1$ & $1,661.9$ \\
\hline & \multicolumn{5}{|c|}{ (In percent of GDP) } \\
\hline $\begin{array}{l}\text { Total expenditure } \\
\text { Expenditure on cash basis }\end{array}$ & $\begin{array}{l}21.2 \\
11.9\end{array}$ & $\begin{array}{l}22.0 \\
11.1\end{array}$ & $\begin{array}{l}24.0 \\
12.1\end{array}$ & $\begin{array}{r}26.6 \\
9.6\end{array}$ & $\begin{array}{r}18.2 \\
7.6\end{array}$ \\
\hline $\begin{array}{l}\text { Current expenditure (excluding } \\
\text { interest arrears) } \\
\text { Defense } \\
\text { Wages and salaries } \\
\text { Development expenditure } \\
\text { Interest arrears }\end{array}$ & $\begin{array}{l}9.0 \\
2.9 \\
1.9 \\
1.7 \\
9.4\end{array}$ & $\begin{array}{r}9.1 \\
3.0 \\
1.6 \\
2.0 \\
10.9\end{array}$ & $\begin{array}{r}9.0 \\
2.9 \\
1.4 \\
1.6 \\
12.0\end{array}$ & $\begin{array}{r}7.1 \\
0.9 \\
2.2 \\
0.6 \\
17.1\end{array}$ & $\begin{array}{r}7.0 \\
1.0 \\
2.1 \\
0.6 \\
10.6\end{array}$ \\
\hline
\end{tabular}

Source: Ministry of Finance and Economic Planning; and Fund staff estimates.

$1 /$ As reported by the authorities.

2/ General social support, including support for poor families, medicine, and medical treatment. 
Table 18. Sudan: Monetary Authorities' Accounts, 1993-November 1997

\begin{tabular}{|c|c|c|c|c|c|}
\hline & 1993 & 1994 & 1995 & 1996 & $\frac{\text { Nov. }}{1997}$ \\
\hline & \multicolumn{5}{|c|}{ (In billions of Sudanese pounds) } \\
\hline Net international reserves & -666 & $-1,343$ & $-2,716$ & $-4,719$ & $-5,334$ \\
\hline Foreign assets & 8 & 31 & 86 & 155 & 129 \\
\hline Foreign liabilities & 674 & 1,374 & 2,798 & 4,874 & 5,463 \\
\hline Net domestic assets & 120 & 213 & 385 & 730 & 821 \\
\hline Net domestic credit & 138 & 168 & 258 & 487 & 605 \\
\hline Net claims on government & 131 & 162 & 252 & 481 & 564 \\
\hline Net lending to government & 140 & 171 & 265 & 589 & 664 \\
\hline Government deposits with BOS & 9 & 9 & 12 & 108 & 100 \\
\hline Central Bank credit to banks & 5 & 4 & 4 & 4 & 39 \\
\hline Central Bank credit to public enterprises & 2 & 2 & 2 & 2 & 2 \\
\hline Other items (net) & -18 & 45 & 127 & 243 & 216 \\
\hline Counterpart to valuation changes & 690 & 1332 & 2,685 & 4,638 & 5,314 \\
\hline Monetary base & 144 & 202 & 357 & 649 & 801 \\
\hline Currency outside banks & 95 & 148 & 249 & 444 & 548 \\
\hline Banks' reserves & 46 & 50 & 97 & 186 & 236 \\
\hline Required reserves & 19 & 28 & 40 & 73 & 117 \\
\hline Excess reserves & 28 & 22 & 58 & 113 & 119 \\
\hline Other deposits with the Central Bank & 4 & 5 & 12 & 19 & 17 \\
\hline
\end{tabular}

Source: Bank of Sudan; and Fund staff projections. 
Table 19. Sudan: Consolidated Balance Sheet of the

Commercial and Specialized Banks, 1993/94-97 1/

(In billions of Sudanese pounds)

\begin{tabular}{|c|c|c|c|c|c|}
\hline & $1993 / 94$ & $1994 / 95$ & 1995 & 1996 & $\frac{\text { Nov. }}{1997}$ \\
\hline Assets & 226.3 & 308.2 & 345.6 & 687.2 & $1,104.5$ \\
\hline Reserves & 57.1 & 79.0 & 80.1 & 134.1 & 259.0 \\
\hline Vault cash & 13.7 & 21.7 & 22.5 & 38.6 & 60.5 \\
\hline Balances with Bank of Sudan & 42.7 & 56.9 & 57.3 & 94.7 & 196.4 \\
\hline Statutory reserves & 22.0 & 35.2 & 39.7 & 44.8 & 116.8 \\
\hline Other balances & 20.7 & 21.7 & 17.6 & 49.9 & 79.6 \\
\hline Other claims & 0.7 & 0.3 & 0.2 & 0.8 & 2.2 \\
\hline Foreign assets, of which: & 98.4 & 119.1 & 168.7 & 276.6 & 431.4 \\
\hline Claims on foreign banks & 86.2 & 109.4 & 153.2 & 244.1 & 391.7 \\
\hline Claims on Central Government & 2.6 & 0.4 & 1.6 & 0.9 & 0.3 \\
\hline Claims on public enterprises & 5.4 & 8.3 & 11.9 & 18.3 & 17.7 \\
\hline Claims on private sector, of which: & 59.9 & 100.0 & 130.7 & 251.8 & 389.3 \\
\hline Agriculture & 16.6 & 19.3 & 30.4 & 60.2 & 107.4 \\
\hline Industry & 11.2 & 18.3 & 25.4 & 49.8 & 75.0 \\
\hline Local trade financing & 5.0 & 4.3 & 3.7 & 10.3 & 20.2 \\
\hline $\begin{array}{l}\text { Claims on nonbank financial } \\
\text { institutions }\end{array}$ & 2.8 & 1.3 & 1.2 & 3.8 & 5.0 \\
\hline Liabilities & 226.3 & 308.2 & 345.6 & 687.2 & $1,104.5$ \\
\hline Demand deposits & 72.3 & 124.5 & 156.0 & 262.0 & 374.9 \\
\hline Time and saving deposits & 13.1 & 20.6 & 26.4 & 39.7 & 107.0 \\
\hline Margin accounts on trade financing & 4.6 & 9.2 & 7.9 & 5.9 & 11.5 \\
\hline Restricted deposits & 0.2 & 0.3 & 0.1 & 0.1 & 0.6 \\
\hline Central Government deposits & 2.9 & 5.9 & 3.1 & 4.6 & 5.0 \\
\hline Government lending funds $2 /$ & 0.2 & 0.0 & 0.0 & 0.0 & 0.0 \\
\hline Liabilities to Bank of Sudan & 1.3 & 6.9 & 7.5 & 3.9 & 0.4 \\
\hline Foreign liabilities, of which: & 15.6 & 19.4 & 28.1 & 42.1 & 44.3 \\
\hline To nonresident banks & 10.7 & 9.3 & 13.4 & 19.8 & 21.3 \\
\hline Foreign currency deposits $3 /$ & 97.5 & 139.9 & 222.9 & 311.3 & 420.2 \\
\hline Capital accounts & 25.1 & 45.0 & 54.7 & 67.3 & 134.5 \\
\hline $\begin{array}{l}\text { Exchange valuation } \\
\text { adiustment (net) 4/ }\end{array}$ & 9.6 & 6.9 & 10.8 & 14.6 & 20.2 \\
\hline $\begin{array}{l}\text { adjustment (net) 4/ } \\
\text { Unclassified liabilities, net 5/ }\end{array}$ & -16.2 & -70.4 & -171.9 & -64.4 & -14.2 \\
\hline \multicolumn{6}{|l|}{ Memorandum item: } \\
\hline Net foreign currency position $6 /$ & -14.8 & -40.2 & -82.3 & -76.8 & -33.1 \\
\hline
\end{tabular}

Source: Bank of Sudan.

1/ Data excludes Agricultural Bank of Sudan.

2/ Funds for onlending in context of Islamic finance.

3 / Foreign currency deposits of Sudanese residents.

4/ Net valuation effect between assets and liabilities.

$5 /$ Net of unclassified assets, including interbranch accounts.

6/ Foreign assets, less foreign liabilities and foreign currency deposits of residents. Small amounts of foreign currency positions may be located elsewhere in the balance sheets. 
Table 20. Sudan: Commercial and Specialized Banks: Balance Sheets Assets and Deposits at Bank of Sudan, September 1997

(In billions of Sudanese pounds)

\begin{tabular}{|c|c|c|}
\hline & $\begin{array}{c}\text { Deposits at } \\
\text { Bank of Sudan }\end{array}$ & $\begin{array}{l}\text { Balance Sheet } \\
\text { Assets }\end{array}$ \\
\hline \multicolumn{3}{|l|}{ Commercial banks } \\
\hline Bank of Khartoum Group 1/ 2/ & 15.4 & 334 \\
\hline El Nilein Bank Group 1/ 3/ & 0.1 & 216 \\
\hline El Tadamon Islamic Bank & 1.9 & 273 \\
\hline Al Shamal Islamic Bank & 0.6 & 96 \\
\hline Sudan Islamic Bank & 0.6 & 49 \\
\hline Sudanese-French Bank & 9.4 & 130 \\
\hline Al Baraka Bank & 0.9 & 52 \\
\hline National Bank of Sudan & 0.1 & 34 \\
\hline Saudi Sudanese Bank & 5.5 & 152 \\
\hline Sudan Commercial Bank 4/ & 0.4 & 58 \\
\hline Faisal Islamic Bank & 3.1 & 75 \\
\hline Omdurman National Bank & 4.1 & 316 \\
\hline El Gharb Islamic Bank & 0.3 & 42 \\
\hline Animal Resources Bank & 0.5 & 48 \\
\hline Citibank & 7.6 & 69 \\
\hline National Bank of Abu Dhabi & 0.6 & 15 \\
\hline Farmers' Bank & 0.3 & 50 \\
\hline Workers National Bank & 0.5 & 18 \\
\hline Islamic Cooperation Development Bank & 0.5 & 46 \\
\hline Al Mashreq Bank & 0.1 & 10 \\
\hline Blue Nile Bank & 0.4 & 6 \\
\hline National Development Bank & 0.6 & 2 \\
\hline Habib Bank & 0.6 & 7 \\
\hline Safa Investment and Credit Bank & 5.5 & 56 \\
\hline Ivory Bank (established 1994) & 0.5 & 4 \\
\hline \multicolumn{3}{|l|}{ Specialized banks } \\
\hline Agricultural Bank of Sudan 1/ & 0.3 & 0 \\
\hline Sudan Savings Bank 1/ & 0.2 & 30 \\
\hline Sudan Estates Bank 1/ & 0.5 & 28 \\
\hline
\end{tabular}

Source: Bank of Sudan.

1/ Government-owned bank.

2/ Merger of Bank of Khartoum, Unity Bank, and Exim Bank in 1993.

3/ Merger of El Nilein Bank and Industrial Bank in 1993.

4/ Privatized in sale to Farmers' Bank.

CInternational Monetary Fund. Not for Redistribution 
Table 21. Sudan: Claims of the Banking System on the Central

Government, 1993/94-97

(In billions of Sudanese pounds)

\begin{tabular}{|c|c|c|c|c|c|}
\hline & 1993/94 & $1994 / 95$ & 1995 & 1996 & $\frac{1997}{\text { Nov. }}$ \\
\hline \multicolumn{6}{|l|}{ Total net claims on } \\
\hline Central Government & 142.9 & 160.2 & 251.0 & 416.7 & 559.0 \\
\hline \multicolumn{6}{|l|}{ Bank of Sudan } \\
\hline Net claims on Central Government & 143.5 & 165.8 & 252.5 & 420.4 & 563.7 \\
\hline Claims on Central Government & 146.9 & 174.3 & 264.9 & 491.4 & 664.2 \\
\hline Main government account & 61.3 & 61.3 & 61.3 & 61.3 & 61.3 \\
\hline Temporary advances & 74.5 & 101.9 & 192.5 & 418.9 & 591.7 \\
\hline Long-term loans & 9.3 & 9.3 & 9.3 & 9.3 & 9.3 \\
\hline Arrears on Fund charges & 0.7 & 0.7 & 0.7 & 0.7 & 0.7 \\
\hline Treasury IMF position & 1.2 & 1.2 & 1.2 & 1.2 & 1.2 \\
\hline Less:Government deposits & 3.4 & 8.5 & 12.4 & 70.9 & 100.5 \\
\hline \multicolumn{6}{|l|}{ Commercial banks } \\
\hline Net claims on Central Government & -0.6 & -5.6 & -1.5 & -3.7 & -4.7 \\
\hline Claims on Central Government & 2.6 & 0.4 & 1.6 & 0.9 & 0.3 \\
\hline $\begin{array}{l}\text { Less:Government deposits, of which: } \\
\text { Of which }\end{array}$ & 2.9 & 6.0 & 3.1 & 4.6 & 5.0 \\
\hline Demand deposits & 2.3 & 2.3 & 2.1 & 3.5 & 0.7 \\
\hline Margin accounts $1 /$ & 0.1 & 0.6 & 0.8 & 0.1 & 0.2 \\
\hline
\end{tabular}

Source: Bank of Sudan.

1/ Margins collected for import transactions. 
Table 22. Sudan: Commercial Banks' Rates of Return and Charge, 1983-97 1/

(In percent per annum or percent ranges)

Jan. 1983- $\quad \underline{\text { April }} \quad \underline{\text { May }} \quad \underline{\text { July }} \quad \underline{\text { July }}$

\begin{tabular}{|c|c|c|}
\hline Sept. 1984 & 1989 & 1990 \\
\hline
\end{tabular}

Lending rates

Discount of bills of exchange

Advances against bills of exchange

Advances to industrial borrowers

Overdrafts and other advances

Murabaha

Short-term 3/

Long-term

By purpose:

Agriculture

Exports

Other productive sectors

Crafts and Cooperatives

Local trade

Musharaka

Minimum on customer participation

Share for management

Bank's share

$\begin{array}{ccc}20 & \ldots & \ldots \\ & \ldots & \ldots \\ 20 & \ldots & \ldots \\ 17 & \ldots & \ldots \\ 17-20 & \ldots & \ldots \\ & & \\ \ldots & 2-3 & 1-3 \\ \ldots & 15-35 & 15-18\end{array}$

Deposit rates

Demand deposits

Fixed deposits

6 months

12 months

Savings deposits

(1)

5-18

$\begin{array}{cccc}30 & 30 & 35 & 45 \\ 30 & 30 & 40 & 45 \\ 36 & 15 & 20 & 40 \\ 25 & 25 & 30 & 40 \\ 48 & 36 & 40 & \ldots\end{array}$

$\cdots$

...

$\begin{array}{llrllll}\ldots & \ldots & 5-25 & \ldots & 55 & 70 & 80\end{array}$

$\quad 20-30 \quad 15-45$

$\quad \ldots \quad 55-85$

... $\quad$.

...

$\cdots$

$\cdots$

$\ldots$

$\cdots$

$\cdots$

$\cdots$

$$
0
$$

0

0

0

14

15

13

Foreign currency deposits

(U.S. dollars and pounds sterling)

Linked to rates in international markets

Source: Bank of Sudan.

1/ The charging and payment of interest was prohibited by law effective September 1984, when banks' operations were made subject to Islamic principles.Observations for April 1989 and later are based on Bank of Sudan circulars.

$2 /$ Minimum annualized nominal rates. Owing to discounts, advance payments, andother lending practices, effective rates are higher.

3/ Monthly profit margins for loans under one year. 
Table 23. Sudan: Commodity Composition of Exports, f.o.b., 1993/94-97

(Value in millions of U.S. dollars; volume in thousand of tons except

when indicated; price in U.S. dollars per ton except when indicated)

\begin{tabular}{|c|c|c|c|c|c|}
\hline & $1993 / 94$ & $1994 / 95$ & 1995 & 1996 & 1997 \\
\hline Total exports & 502.6 & 421.7 & 555.6 & 620.2 & 579.8 \\
\hline Total recorded exports & 500.0 & 421.5 & 555.6 & 620.2 & 579.8 \\
\hline Cotton & 95.9 & 95.5 & 123.0 & 128.2 & 111.2 \\
\hline Extra long staple & 35.9 & 26.4 & 31.7 & 25.9 & 18.9 \\
\hline Volume (thousand bales) & 134.4 & 71.9 & 86.1 & 50.9 & 46.0 \\
\hline Unit value (U.S. cents/lb) & 63.6 & 87.4 & 87.5 & 121.2 & 98.0 \\
\hline Other & 60.0 & 69.1 & 91.3 & 102.3 & 92.2 \\
\hline Volume (thousand bales) & 276.2 & 244.1 & 348.6 & 383.2 & 410.0 \\
\hline Unit value (U.S. cents/lb) & 51.7 & 67.4 & 62.4 & 63.6 & 53.6 \\
\hline Groundnuts & 25.8 & 23.1 & 21.2 & 22.8 & 67.8 \\
\hline Seeds and edible nuts & 4.6 & 3.0 & 2.5 & 1.2 & 6.6 \\
\hline Volume & 12.3 & 7.0 & 4.5 & 2.1 & 12.1 \\
\hline Unit value & 375.2 & 428.6 & 555.6 & 571.4 & 548.4 \\
\hline Oil & 11.7 & 14.0 & 11.5 & 12.6 & 46.8 \\
\hline Volume & 17.1 & 21.3 & 12.5 & 14.0 & 54.4 \\
\hline Unit value & 685.1 & 657.3 & 920.0 & 900.0 & 860.8 \\
\hline Cake & 9.5 & 6.1 & 7.2 & 9.0 & 14.3 \\
\hline Volume & 75.2 & 57.8 & 64.7 & 67.5 & 86.4 \\
\hline Unit value & 126.3 & 105.5 & 111.3 & 133.3 & 165.2 \\
\hline Sesame & 76.7 & 60.6 & 80.6 & 141.1 & 117.0 \\
\hline Seed & 74.2 & 60.6 & 80.3 & 141.0 & 116.1 \\
\hline Volume & 137.3 & 91.9 & 97.4 & 157.4 & 172.1 \\
\hline Unit value & 540.3 & 659.4 & 824.4 & 895.8 & 674.6 \\
\hline Cake & 2.5 & 0.0 & 0.3 & 0.1 & 0.9 \\
\hline Volume & 15.2 & 3.0 & 1.7 & 0.9 & 4.6 \\
\hline Unit value & 164.5 & - & 176.5 & 111.1 & 192.9 \\
\hline Dura & 34.9 & 21.4 & 44.0 & 2.6 & 0.0 \\
\hline Volume & 358.9 & 210.5 & 407.0 & 17.3 & 0.0 \\
\hline Unit value & 97.3 & 101.7 & 108.1 & 150.3 & 0.0 \\
\hline Gum arabic & 81.0 & 44.7 & 51.4 & 29.2 & 23.1 \\
\hline Volume & 24.1 & 15.3 & 18.5 & 15.3 & 20.0 \\
\hline Unit value & $3,361.0$ & $2,923.7$ & $2,778.5$ & $1,908.5$ & $1,158.2$ \\
\hline Sugar & 32.8 & 15.8 & 22.0 & 33.1 & 28.7 \\
\hline Volume & 178.8 & 128.1 & 215.8 & 279.4 & 125.7 \\
\hline Unit value & 183.2 & 123.3 & 101.9 & 118.5 & 228.7 \\
\hline Livestock & 89.8 & 61.7 & 84.1 & 81.2 & 73.1 \\
\hline Sheep and lambs & 76.8 & 57.5 & 71.6 & 74.5 & 69.4 \\
\hline Volume (thousand head) & 793.0 & 707.8 & 884.4 & $1,187.6$ & $1,172.9$ \\
\hline Unit value (U.S. dollars per head) & 96.8 & 81.2 & 80.9 & 62.7 & 59.1 \\
\hline Cattle & 4.7 & 0.2 & 1.0 & 2.5 & 0.4 \\
\hline Camels and goats & 8.3 & 4.0 & 11.5 & 4.2 & 3.4 \\
\hline Other & 63.1 & 98.7 & 129.4 & 182.0 & 158.9 \\
\hline Hibiscus (karkadeh) & 15.2 & 11.8 & 13.1 & 13.4 & 16.8 \\
\hline Yam & 0.5 & 2.9 & 7.2 & 15.1 & 10.4 \\
\hline Watermelon seeds & 8.3 & 6.6 & 8.1 & 22.5 & 15.3 \\
\hline Hides and skins & 9.4 & 15.1 & 20.5 & 28.7 & 19.3 \\
\hline Fruits and vegetables & 2.1 & 2.5 & 4.4 & 3.4 & 2.6 \\
\hline Gold & 22.0 & 16.8 & 46.7 & 53.3 & 45.6 \\
\hline Other & 5.6 & 43.0 & 29.4 & 45.6 & 48.8 \\
\hline Unrecorded in customs data & 2.6 & 0.2 & 0.0 & 0.0 & 0.0 \\
\hline Livestock & 0.0 & 0.0 & 0.0 & 0.0 & 0.0 \\
\hline Canmels (Egypt bilateral) & 2.6 & 0.2 & 0.0 & 0.0 & 0.0 \\
\hline
\end{tabular}

Sources: Information provided by the Sudanese authorities; and Fund staff estimates. 
Table 24. Sudan: Commodity Composition of Imports, c.i.f., 1993/94-97

(In millions of U.S. dollar)

\begin{tabular}{|c|c|c|c|c|c|}
\hline & $1993 / 94$ & $1994 / 95$ & 1995 & 1996 & 1997 \\
\hline Total Imports & $1,227.4$ & $1,023.4$ & $1,218.8$ & $1,547.3$ & $1,554.9$ \\
\hline Foodstuffs $1 /$ & 204.8 & 181.4 & 214.5 & 232.2 & 242.8 \\
\hline Wheat & 9.6 & 5.7 & 30.3 & 47.5 & 72.5 \\
\hline Wheat flour & 55.8 & 40.0 & 58.9 & 50.3 & 60.4 \\
\hline Sugar & 0.0 & 0.1 & 0.8 & 0.5 & 0.0 \\
\hline Tea & 32.1 & 26.9 & 26.6 & 33.4 & 31.8 \\
\hline Coffee & 8.5 & 5.1 & 9.0 & 12.9 & 17.7 \\
\hline Dairy products & 2.2 & 8.1 & 9.1 & 6.3 & 8.4 \\
\hline Animal and vegetable oils & 7.0 & 25.6 & 5.8 & 10.9 & 1.6 \\
\hline Other foodstuffs & 89.5 & 69.9 & 74.0 & 70.4 & 50.6 \\
\hline Customs data & 39.1 & 40.1 & 38.2 & 42.9 & 35.6 \\
\hline World Food Program & 50.4 & 29.8 & 35.8 & 27.5 & 15.0 \\
\hline Drinks and tobacco & 7.9 & 14.5 & 16.5 & 15.2 & 17.3 \\
\hline Crude materials & 254.5 & 196.8 & 224.8 & 354.8 & 331.7 \\
\hline Petroleum & 230.4 & 178.2 & 194.2 & 305.9 & 299.4 \\
\hline Customs data & 230.4 & 178.2 & 194.2 & 305.9 & 299.4 \\
\hline Quantity & $1,372.1$ & $1,032.2$ & $1,162.4$ & $1,489.2$ & $1,633.0$ \\
\hline Unit value & 169.8 & 169.8 & 165.3 & 205.5 & 183.7 \\
\hline GPC petroleum & 0.0 & 0.0 & 0.0 & 0.0 & 0.0 \\
\hline Other & 24.1 & 18.6 & 30.6 & 48.9 & 32.4 \\
\hline Chemicals & 113.3 & 116.8 & 134.0 & 233.7 & 191.7 \\
\hline Pharmaceuticals & 3.0 & 40.4 & 38.1 & 28.6 & 33.7 \\
\hline Fertilizers & 29.1 & 11.0 & 15.7 & 74.8 & 52.4 \\
\hline Insecticides & 5.6 & 25.0 & 31.8 & 6.9 & 3.3 \\
\hline Other & 30.8 & 40.4 & 48.2 & 123.4 & 102.3 \\
\hline Manufactured goods & 293.2 & 228.6 & 276.6 & 298.3 & 285.1 \\
\hline Machinery, equipment & 202.1 & 188.5 & 209.8 & 253.8 & 261.4 \\
\hline Transport equipment & 134.2 & 70.4 & 112.4 & 133.3 & 170.7 \\
\hline Automobiles & 15.3 & 18.2 & 38.1 & 24.1 & 24.3 \\
\hline Trucks & 46.5 & 8.9 & 18.0 & 51.2 & 39.1 \\
\hline Auto spare parts & 25.9 & 21.8 & 17.8 & 21.5 & 65.7 \\
\hline Other & 17.1 & 21.5 & 38.5 & 36.5 & 41.6 \\
\hline Textiles & 17.4 & 26.3 & 30.2 & 26.1 & 54.1 \\
\hline
\end{tabular}

Sources: Information provided by the Sudanese authorities; and Fund staff estimates.

1/ Includes commodity aid imports, and emergency aid assistance. 
Table 25. Sudan: Summary Index of Export and Import Prices and Volumes, 1993/94-97

(Index 1993/94 = 100)

\begin{tabular}{|c|c|c|c|c|c|}
\hline & 1993/94 & $1994 / 95$ & 1995 & 1996 & 1997 \\
\hline Total Exports 1/ & 100.0 & 83.9 & 110.5 & 123.4 & 115.3 \\
\hline Change in percent & 43.1 & -16.1 & 31.8 & 11.6 & -6.5 \\
\hline Export volume & 100.0 & 70.5 & 90.2 & 96.8 & 109.3 \\
\hline Change in percent & 16.1 & -29.5 & 27.9 & 7.3 & 12.9 \\
\hline Export prices & 100.0 & 119.0 & 122.6 & 127.6 & 105.7 \\
\hline Change in percent & 23.2 & 19.0 & 3.0 & 4.1 & -17.2 \\
\hline Total Imports & 100.0 & 83.4 & 99.3 & 126.1 & 126.7 \\
\hline Change in percent & 14.9 & -16.6 & 19.1 & 27.0 & 0.5 \\
\hline Import volume & 100.0 & 78.6 & 90.9 & 110.0 & 120.6 \\
\hline Change in percent & 17.0 & -21.4 & 15.7 & 20.9 & 9.7 \\
\hline Import prices & 100.0 & 106.1 & 109.2 & 114.6 & 105.0 \\
\hline Change in percent & -1.8 & 6.1 & 2.9 & 5.0 & -8.4 \\
\hline Terms of trade & 100.0 & 112.1 & 112.2 & 111.2 & 100.5 \\
\hline Change in percent & 25.5 & 12.1 & 0.1 & -0.9 & -9.6 \\
\hline
\end{tabular}

Source: Staff estimates based on data provided by the Sudanese authorities.

1/ Excluding unclassified exports. 
Table 26. Sudan: Destination of Exports, 1992-96

(In percent of total)

\begin{tabular}{|c|c|c|c|c|c|}
\hline & 1992 & 1993 & 1994 & 1995 & 1996 \\
\hline Industrial countries & 36.6 & 38.7 & 42.2 & 46.1 & 40.2 \\
\hline \multicolumn{6}{|l|}{ Of which } \\
\hline United States & 1.3 & 1.8 & 3.4 & 4.7 & 2.1 \\
\hline France & 6.5 & 3.6 & 5.5 & 3.0 & 1.4 \\
\hline Germany & 5.0 & 4.6 & 2.7 & 3.9 & 3.1 \\
\hline Italy & 9.0 & 4.7 & 7.6 & 8.0 & 7.7 \\
\hline Japan & 5.0 & 6.5 & 4.5 & 6.2 & 4.0 \\
\hline United Kingdom & 4.9 & 9.0 & 7.4 & 14.3 & 12.6 \\
\hline African countries & 2.5 & 2.2 & 4.2 & 1.7 & 2.8 \\
\hline Asian countries & 10.1 & 7.4 & 17.4 & 19.5 & 17.9 \\
\hline \multicolumn{6}{|l|}{ Ofwhich } \\
\hline China & 0.1 & 0.1 & 6.1 & 10.8 & 6.8 \\
\hline Thailand & 6.0 & 0.2 & 4.6 & 3.9 & 7.6 \\
\hline South Korea & 0.4 & 0.2 & 1.7 & 0.9 & 1.4 \\
\hline Taiwan & 0.1 & 6.0 & 0.1 & 0.2 & 0.1 \\
\hline Middle East countries & 49.4 & 40.0 & 35.1 & 32.5 & 37.3 \\
\hline \multicolumn{6}{|l|}{ Ofwhich } \\
\hline Egypt & 7.4 & 2.0 & 0.7 & 4.7 & 6.7 \\
\hline Saudi Arabia & 19.3 & 25.2 & 23.2 & 19.5 & 18.9 \\
\hline United Arab Emirates & 0.7 & 0.7 & 0.4 & 1.5 & 1.9 \\
\hline Libya & 11.7 & 4.2 & 4.5 & 1.2 & 0.7 \\
\hline Other countries & 1.4 & 11.7 & 1.1 & 0.2 & 1.8 \\
\hline Total & 100.0 & 100.0 & 100.0 & 100.0 & 100.0 \\
\hline
\end{tabular}

Source: Fund staff estimates based on Bank of Sudan, Foreign Trade Statistical Digest-several issues. 
Table 27. Sudan: Sources of Imports, 1992-96

(In percent of total)

\begin{tabular}{|c|c|c|c|c|c|}
\hline & 1992 & 1993 & 1994 & 1995 & 1996 \\
\hline $\begin{array}{l}\text { Industrial countries } \\
\text { Of which: }\end{array}$ & 36.0 & 44.5 & 40.6 & 44.2 & 38.4 \\
\hline United States & 7.9 & 3.4 & 3.5 & 5.0 & 4.6 \\
\hline France & 1.9 & 3.9 & 3.7 & 5.9 & 5.5 \\
\hline Germany & 4.1 & 2.7 & 3.7 & 6.4 & 4.7 \\
\hline Italy & 3.7 & 6.7 & 3.1 & 2.9 & 2.9 \\
\hline United Kingdom & 7.8 & 5.0 & 11.3 & 6.1 & 6.9 \\
\hline Japan & 3.5 & 11.0 & 3.7 & 5.7 & 4.2 \\
\hline $\begin{array}{l}\text { African Countries } \\
\text { Of which: }\end{array}$ & 0.2 & 4.4 & 4.5 & 4.4 & 6.7 \\
\hline Kenya & 0.1 & 2.9 & 1.8 & 1.6 & 1.9 \\
\hline $\begin{array}{l}\text { Asian countries } \\
\text { Of which: }\end{array}$ & 11.1 & 12.8 & 17.7 & 17.5 & 20.6 \\
\hline China & 2.1 & 2.8 & 3.2 & 3.6 & 4.3 \\
\hline South Korea & 0.7 & 1.4 & 1.6 & 6.9 & 1.6 \\
\hline India & 1.4 & 2.0 & 2.0 & 2.1 & 3.8 \\
\hline Indonesia & 1.1 & 2.7 & 2.0 & 1.0 & 1.1 \\
\hline $\begin{array}{l}\text { Middle East Countries } \\
\text { Of which: }\end{array}$ & 43.4 & 30.0 & 28.9 & 24.9 & 22.6 \\
\hline Egypt & 5.2 & 4.9 & 6.0 & 6.1 & 3.5 \\
\hline Saudi Arabia & 21.3 & 15.4 & 16.0 & 10.3 & 11.3 \\
\hline United Arab Emirates & 6.4 & 1.0 & 2.7 & 4.3 & 4.7 \\
\hline Other countries & 9.3 & 8.3 & 8.3 & 9.0 & 11.7 \\
\hline Total & 100.0 & 100.0 & 100.0 & 100.0 & 100.0 \\
\hline
\end{tabular}

Source: Fund staff estimates based on Bank of Sudan, Foreign Trade Statistics Digest-several issu 
Table 28. Sudan: Official Transfers, 1993/94-1997

(In millions of U.S. dollars)

\begin{tabular}{|c|c|c|c|c|c|}
\hline & $1993 / 94$ & $1994 / 95$ & 1995 & 1996 & $\frac{1997}{\text { Prel. }}$ \\
\hline Total official transfers & 88.1 & 52.3 & 54.4 & 48.3 & 19.1 \\
\hline Multilateral creditors & 78.8 & 48.1 & 53.6 & 40.6 & 11.2 \\
\hline Of which project grants & 13.2 & 11.1 & 10.6 & 7.7 & 1.2 \\
\hline Official bilateral creditors & 9.3 & 4.2 & 2.6 & 0.0 & 0.0 \\
\hline Paris Club & 9.3 & 4.4 & 2.6 & 0.0 & 0.0 \\
\hline Non-Paris Club & 0.0 & 0.0 & 0.0 & 0.0 & 0.0 \\
\hline Cash and commodity aid & 69.3 & 40.7 & 43.8 & 32.9 & 15.0 \\
\hline 1. Budgetary inflows & 18.7 & 16.7 & 15.6 & 8.6 & 0.0 \\
\hline Official bilateral & 3.7 & 3.7 & 0.8 & 0.0 & 0.0 \\
\hline France & 0.0 & 0.0 & 0.0 & 0.0 & 0.0 \\
\hline Germany & 0.0 & 0.0 & 0.0 & 0.0 & 0.0 \\
\hline Italy & 3.7 & 3.7 & 0.8 & 0.0 & 0.0 \\
\hline Japan & 0.0 & 0.0 & 0.0 & 0.0 & 0.0 \\
\hline Netherlands & 0.0 & 0.0 & 0.0 & 0.0 & 0.0 \\
\hline Pakistan & 0.0 & 0.0 & 0.0 & 0.0 & 0.0 \\
\hline Multilaterals & 15.0 & 13.0 & 14.8 & 8.6 & 0.0 \\
\hline World Food Program & 15.0 & 13.0 & 14.8 & 8.6 & 0.0 \\
\hline Food for schools, etc. & 12.5 & $\ldots$ & $\ldots$ & $\ldots$ & 0.0 \\
\hline Food for sale & 2.5 & $\cdots$ & $\cdots$ & $\ldots$ & $\begin{array}{l}0.0 \\
0.0\end{array}$ \\
\hline 2. Non-budgetary inflows & 50.6 & 24.0 & 28.2 & 24.3 & 15.0 \\
\hline World Food Program & 50.6 & 24.0 & 28.2 & 24.3 & 15.0 \\
\hline Food aid & $\ldots$ & 16.8 & 21.0 & 18.9 & 15.0 \\
\hline Cash aid & $\ldots$ & 7.2 & 7.2 & 5.4 & 0.0 \\
\hline Project aid & 18.8 & 11.6 & 10.6 & 7.8 & 4.1 \\
\hline 1. Budgetary inflows & 12.8 & 5.9 & 6.0 & 2.9 & 0.1 \\
\hline Official bilateral & 5.6 & 0.5 & 1.8 & 0.0 & 0.0 \\
\hline Canada & 0.0 & 0.0 & 0.0 & 0.0 & 0.0 \\
\hline Germany & 0.6 & 0.7 & 1.8 & 0.0 & 0.0 \\
\hline Iran & 5.0 & 0.0 & 0.0 & 0.0 & 0.0 \\
\hline Japan & 0.0 & 0.0 & 0.0 & 0.0 & 0.0 \\
\hline Saudi Arabia & 0.0 & 0.0 & 0.0 & 0.0 & 0.0 \\
\hline Multilaterals & 7.2 & 5.4 & 4.2 & 2.9 & 0.1 \\
\hline African Development Fund & 2.7 & 2.6 & 2.6 & 2.7 & 0.0 \\
\hline $\mathrm{EEC} / \mathrm{EDF}$ & 4.5 & 2.4 & 1.3 & 0.0 & 0.0 \\
\hline Islamic Development Bank & 0.0 & 0.4 & 0.3 & 0.1 & 0.1 \\
\hline UNDP & 0.0 & 0.0 & 0.0 & 0.0 & 0.0 \\
\hline 2. Non-budgetary inflows & 6.0 & 5.7 & 4.7 & 4.9 & 4.0 \\
\hline Multilaterals & 6.0 & 5.7 & 4.7 & 4.9 & 4.0 \\
\hline UNDP (TA) & 6.0 & 5.7 & 4.7 & 4.9 & 4.0 \\
\hline
\end{tabular}

Sources: Sudanese authorities; and Fund staff estimates. 
Table 29. Sudan: Loan Disbursements, 1993/94-1997

(In millions of U.S. dollars)

\begin{tabular}{|c|c|c|c|c|c|}
\hline & $1993 / 94$ & $1994 / 95$ & 1995 & 1996 & 1997 \\
\hline Total loan disbursements & 77.5 & 76.7 & 86.0 & 28.6 & 16.5 \\
\hline Multilateral creditors & 44.9 & 76.7 & 86.0 & 16.4 & 16.5 \\
\hline Bilateral creditors & 7.6 & 0.0 & 0.0 & 0.0 & 0.0 \\
\hline Paris Club & 0.9 & 0.0 & 0.0 & 0.0 & 0.0 \\
\hline Non-Paris Club & 6.7 & 0.0 & 0.0 & 0.0 & 0.0 \\
\hline Commercial banks & 25.0 & 0.0 & 0.0 & 0.0 & 0.0 \\
\hline Cash and Commodity loans & 45.2 & 20.9 & 22.9 & 12.7 & 0.2 \\
\hline Multilateral Funds/Banks & 0.0 & 20.9 & 22.9 & 0.0 & 0.0 \\
\hline African Development Bank & 0.0 & 7.2 & 22.9 & 0.0 & 0.0 \\
\hline Islamic Development Bank & 0.0 & 0.0 & 0.0 & 0.6 & 0.0 \\
\hline OPEC Fund & 0.0 & 13.7 & 0.0 & 0.0 & 0.2 \\
\hline Other & 45.2 & 0.0 & 0.0 & 0.0 & 0.0 \\
\hline Libyan Arab Foreign Bank & 25.0 & 0.0 & 0.0 & 0.0 & 0.0 \\
\hline Other & 20.2 & 0.0 & 0.0 & 12.1 & 0.0 \\
\hline Project loans (budget) & 52.5 & 55.8 & 63.1 & 15.9 & 16.3 \\
\hline Multilaterals & 44.9 & 55.8 & 63.1 & 15.8 & 16.3 \\
\hline IDA (World Bank) & 33.4 & 0.0 & 0.0 & 0.0 & 0.0 \\
\hline IFAD & 4.3 & 1.0 & 2.5 & 13.4 & 12.5 \\
\hline African Development Bank & 2.9 & 22.9 & 26.2 & 0.0 & 0.0 \\
\hline African Development Fund & 4.1 & 29.2 & 31.5 & 0.1 & 0.0 \\
\hline European Investment Bank & 0.0 & 0.0 & 0.0 & 0.0 & 0.0 \\
\hline Islamic Development Bank & 0.2 & 2.6 & 2.8 & 1.9 & 3.7 \\
\hline OPEC Fund & 0.1 & 0.0 & 0.1 & 0.6 & 0.2 \\
\hline Arab Funds & 0.1 & 0.0 & 0.0 & 0.0 & 0.0 \\
\hline Kuwait Fund & 0.1 & 0.0 & 0.0 & 0.0 & 0.0 \\
\hline Other & 7.5 & 0.0 & 0.0 & 0.0 & 0.0 \\
\hline Germany & 0.9 & 0.0 & 0.0 & 0.0 & 0.0 \\
\hline China & 6.6 & 0.0 & 0.0 & 0.1 & 0.0 \\
\hline
\end{tabular}

Sources: Sudanese authorities; and Fund staff estimates. 
Table 30. Sudan: Total External Arrears, 1994-97

(In millions of U.S. dollars)

\begin{tabular}{|c|c|c|c|c|}
\hline & 1994 & 1995 & 1996 & 1997 \\
\hline Total & $13,738.0$ & $16,310.0$ & $17,217.2$ & $18,482.7$ \\
\hline Multilaterals & $2,277.7$ & $2,373.4$ & $2,432.0$ & $2,504.2$ \\
\hline IMF & $1,736.0$ & $1,763.8$ & $1,697.0$ & $1,662.3$ \\
\hline IBRD group & 32.1 & 27.6 & 74.0 & 97.6 \\
\hline IBRD & 1.0 & 2.3 & 3.0 & 4.1 \\
\hline IDA & 31.1 & 25.3 & 71.0 & 93.5 \\
\hline Arab Monetary Fund & 259.9 & 295.1 & 307.0 & 322.4 \\
\hline Islamic Development Bank & 0.8 & 1.1 & 0.0 & 0.0 \\
\hline African Development Bank & 5.2 & 12.7 & 8.0 & 20.6 \\
\hline African Development Fund & 3.1 & 8.5 & 17.0 & 24.6 \\
\hline Arab Fund for Ec/Soc & $\ldots$ & 192.9 & 239.0 & 268.2 \\
\hline European Investment Bank & 32.6 & 24.8 & 32.0 & 37.6 \\
\hline \multicolumn{5}{|c|}{ International Fund for Agriculture } \\
\hline Development & 0.7 & 0.8 & 1.0 & 2.2 \\
\hline OPEC Fund & 14.9 & 12.0 & 19.0 & 28.8 \\
\hline APICORP & 22.7 & 34.2 & 38.0 & 39.9 \\
\hline Paris Club bilaterals & $3,663.7$ & $4,423.4$ & $4,746.0$ & $5,235.4$ \\
\hline Rescheduled & $2,867.9$ & $3,553.0$ & $3,826.0$ & $4,205.8$ \\
\hline Unrescheduled & 795.8 & 870.4 & 920.0 & $1,029.6$ \\
\hline Non-Paris Club Bilaterals & $4,330.9$ & $5,073.0$ & $5,463.0$ & $5,846.6$ \\
\hline OPEC countries & $\ldots$ & $4,459.0$ & $4,869.0$ & $5,197.9$ \\
\hline Eastern European countries & $\ldots$ & 359.0 & 337.0 & 356.5 \\
\hline Other countries & $\ldots$ & 255.0 & 257.0 & 292.1 \\
\hline Commercial & $3,465.7$ & $4,440.3$ & $4,576.2$ & $4,896.5$ \\
\hline Banks & $2,823.7$ & $3,640.6$ & $3,569.5$ & $3,819.3$ \\
\hline Suppliers & 642.0 & 799.6 & $1,006.7$ & $1,077.2$ \\
\hline \multicolumn{5}{|l|}{ Memorandum items: } \\
\hline \multicolumn{5}{|l|}{ Proportion of total debt in arrears } \\
\hline Total & 80.3 & 82.4 & 83.9 & 85.7 \\
\hline Multilaterals & 57.9 & 56.2 & 56.8 & 57.9 \\
\hline Paris Club & 78.8 & 82.6 & 86.0 & 88.7 \\
\hline Non-Paris Club bilats & 85.7 & 87.8 & 89.0 & 90.7 \\
\hline Commercial & 99.9 & 100.0 & 100.0 & 100.0 \\
\hline
\end{tabular}

Sources: Bank of Sudan and Fund staff estimates. 
Table 31. Sudan: Breakdown of Paris Club Debt, 1993-97

(In millions of U.S. dollars)

\begin{tabular}{|c|c|c|c|c|c|}
\hline & 1993 & 1994 & 1995 & 1996 & 1997 \\
\hline Total & $4,447.9$ & $4,649.6$ & $4,981.8$ & $5,520.8$ & $5,843.1$ \\
\hline Austria & 383.9 & 402.2 & 432.1 & 497.1 & 524.3 \\
\hline Belgium & 255.3 & 267.8 & 288.0 & 354.7 & 374.8 \\
\hline Canada & 18.7 & 19.5 & 20.9 & 24.4 & 26.9 \\
\hline Denmark & 137.5 & 143.6 & 153.7 & 171.0 & 180.2 \\
\hline France & 573.2 & 599.2 & 641.9 & 724.6 & 764.1 \\
\hline Germany & 122.2 & 128.0 & 137.5 & 188.5 & 198.2 \\
\hline Ireland & 4.4 & 4.6 & 5.0 & 7.6 & 8.0 \\
\hline Italy & 471.9 & 493.8 & 529.7 & 572.7 & 604.3 \\
\hline Japan & 388.7 & 405.9 & 434.5 & 422.9 & 446.9 \\
\hline Netherlands & 193.6 & 203.1 & 218.6 & 246.6 & 260.5 \\
\hline Norway & 44.5 & 46.3 & 49.3 & 72.3 & 76.2 \\
\hline Spain & 25.6 & 26.6 & 28.2 & 31.1 & 32.9 \\
\hline Switzerland & 126.6 & 132.6 & 142.5 & 165.6 & 175.2 \\
\hline United Kingdom & 617.2 & 647.5 & 696.7 & 834.2 & 878.8 \\
\hline United States & $1,084.6$ & $1,128.8$ & $1,203.1$ & $1,270.6$ & $1,291.8$ \\
\hline Previously Rescheduled Debt & $3,194.8$ & $3,351.7$ & $3,606.6$ & $4,122.7$ & $4,352.4$ \\
\hline Austria & 344.7 & 361.6 & 389.1 & 457.9 & 483.1 \\
\hline Belgium & 250.3 & 262.6 & 282.6 & 347.9 & 367.2 \\
\hline Canada & 11.4 & 12.0 & 12.9 & 13.9 & 15.8 \\
\hline Denmark & 90.4 & 94.8 & 102.1 & 123.9 & 130.8 \\
\hline France & 409.2 & 429.3 & 461.9 & 547.2 & 577.9 \\
\hline Germany & 108.5 & 113.8 & 122.5 & 174.8 & 183.8 \\
\hline Ireland & 4.4 & 4.6 & 5.0 & 7.6 & 8.0 \\
\hline Italy & 376.1 & 394.6 & 424.6 & 479.5 & 506.4 \\
\hline Japan & 250.8 & 263.1 & 283.1 & 291.6 & 307.6 \\
\hline Netherlands & 193.6 & 203.1 & 218.6 & 246.6 & 260.5 \\
\hline Norway & 15.3 & 16.1 & 17.3 & 26.7 & 28.3 \\
\hline Spain & 4.2 & 4.4 & 4.7 & 5.1 & 5.4 \\
\hline Switzerland & 112.9 & 118.4 & 127.5 & 149.5 & 158.3 \\
\hline United Kingdom & 615.2 & 645.4 & 694.5 & 832.2 & 876.7 \\
\hline United States & 407.8 & 427.8 & 460.4 & 418.3 & 442.7 \\
\hline Debt Not Previously Rescheduled 1/ & $1,253.1$ & $1,297.9$ & $1,375.1$ & $1,398.1$ & $1,490.7$ \\
\hline Austria & 39.2 & 40.6 & 43.0 & 39.2 & 41.2 \\
\hline Belgium & 5.0 & 5.2 & 5.5 & 6.8 & 7.6 \\
\hline Canada & 7.3 & 7.6 & 8.0 & 10.5 & 11.1 \\
\hline Denmark & 47.1 & 48.8 & 51.7 & 47.1 & 49.5 \\
\hline France & 164.0 & 169.9 & 180.0 & 177.4 & 186.3 \\
\hline Germany & 13.7 & 14.2 & 15.0 & 13.7 & 14.4 \\
\hline Ireland & 0.0 & 0.0 & 0.0 & 0.0 & 0.0 \\
\hline Italy & 95.8 & 99.2 & 105.1 & 93.2 & 97.9 \\
\hline Japan & 137.9 & 142.8 & 151.3 & 131.3 & 139.4 \\
\hline Netherlands & 0.0 & 0.0 & 0.0 & 0.0 & 0.0 \\
\hline Norway & 29.2 & 30.2 & 32.0 & 45.6 & 47.9 \\
\hline Spain & 21.4 & 22.2 & 23.5 & 26.0 & 27.5 \\
\hline Switzerland & 13.7 & 14.2 & 15.0 & 16.1 & 16.9 \\
\hline United Kingdom & 2.0 & 2.1 & 2.2 & 2.0 & 2.1 \\
\hline United States & 676.8 & 701.0 & 742.7 & 789.3 & 849.1 \\
\hline
\end{tabular}

Source: Staff estimates based on information provided by the Sudanese authorities.

1/ Breakdown between pre- and post-cut off date debt not provided. 
Table 32. Sudan: External Public Debt (including arrears), 1994-97 (In millions of U.S. dollars)

\begin{tabular}{|c|c|c|c|c|}
\hline & 1994 & 1995 & 1996 & 1997 \\
\hline Total & $17,104.8$ & $19,798.3$ & $20,516.2$ & $21,566.3$ \\
\hline Multilaterals & $3,934.9$ & $4,223.5$ & $4,282.0$ & $4,322.9$ \\
\hline IMF & $1,736.0$ & $1,763.8$ & $1,697.0$ & $1,662.3$ \\
\hline IBRD group & $1,141.9$ & $1,287.8$ & $1,282.0$ & $1,291.8$ \\
\hline IBRD & 6.3 & 6.8 & 6.0 & 6.3 \\
\hline IDA & $1,135.7$ & $1,281.0$ & $1,276.0$ & $1,285.5$ \\
\hline Arab Monetary Fund & 259.9 & 295.1 & 307.0 & 322.4 \\
\hline Islamic Development Bank & 10.3 & 11.2 & 13.0 & 16.7 \\
\hline African Development Bank & 50.2 & 81.3 & 90.0 & 96.6 \\
\hline African Development Fund & 239.3 & 272.5 & 353.0 & 355.6 \\
\hline Arab Fund for Ec/Soc & 263.2 & 278.7 & 296.0 & 316.1 \\
\hline European Investment Bank & 78.1 & 71.7 & 67.0 & 68.3 \\
\hline \multicolumn{5}{|c|}{ International Fund for Agriculture } \\
\hline Development & $\ldots$ & 73.5 & 84.0 & 95.5 \\
\hline OPEC Fund & 47.5 & 53.8 & 55.0 & 57.8 \\
\hline APICORP & 26.8 & 34.2 & 38.0 & 39.9 \\
\hline Paris Club bilaterals & $4,648.7$ & $5,354.6$ & $5,521.0$ & $5,900.2$ \\
\hline Rescheduled & $3,351.7$ & $3,979.5$ & $4,122.0$ & $4,414.8$ \\
\hline Unrescheduled & $1,297.0$ & $1,375.1$ & $1,399.0$ & $1,485.4$ \\
\hline Non-Paris Club Bilaterals & $5,052.5$ & $5,781.0$ & $6,137.0$ & $6,446.6$ \\
\hline OPEC countries & $4,253.1$ & $4,975.0$ & $5,385.0$ & $5,659.9$ \\
\hline Eastern European countries & 385.3 & 351.0 & 337.0 & 356.5 \\
\hline Other countries & 414.1 & 455.0 & 415.0 & 430.1 \\
\hline Commercial & $3,468.7$ & $4,439.3$ & $4,576.2$ & $4,896.5$ \\
\hline Banks & $2,826.7$ & $3,639.6$ & $3,569.5$ & $3,819.3$ \\
\hline Suppliers & 642.0 & 799.6 & $1,006.7$ & $1,077.2$ \\
\hline
\end{tabular}

Sources: Bank of Sudan and Fund staff estimates. 
Table 33. Sudan: External Public Debt Service Obligations, 1993/94-97

(In millions of U.S. dollars)

\begin{tabular}{|c|c|c|c|c|c|}
\hline & 1993/94 & $1994 / 95$ & 1995 & 1996 & 1997 \\
\hline Principal due & 374.0 & 311.8 & 287.6 & 279.6 & 232.9 \\
\hline IMF & 0.0 & 0.0 & 0.0 & 0.0 & 0.0 \\
\hline Other & 374.0 & 311.8 & 287.6 & 279.6 & 232.9 \\
\hline Interest due & 868.3 & 931.2 & 912.6 & 956.2 & $1,107.8$ \\
\hline IMF 1/ & 54.7 & 49.7 & 53.1 & 40.2 & 37.0 \\
\hline Other $2 /$ & 813.6 & 881.4 & 859.6 & 915.9 & $1,070.8$ \\
\hline Total debt service due & $1,242.4$ & $1,243.0$ & $1,200.3$ & $1,235.7$ & $1,340.7$ \\
\hline IMF 1/ & 54.7 & 49.7 & 53.1 & 40.2 & 37.0 \\
\hline Other $2 /$ & $1,187.6$ & $1,193.2$ & $1,147.2$ & $1,195.5$ & $1,303.7$ \\
\hline Actual debt service payments & 30.6 & 68.4 & 73.5 & 56.2 & 61.5 \\
\hline IMF 3/ & 5.3 & 53.4 & 55.6 & 49.9 & 30.2 \\
\hline Other & 25.4 & 15.0 & 17.9 & 6.3 & 1.3 \\
\hline \multicolumn{6}{|l|}{ Of which: } \\
\hline \multicolumn{6}{|l|}{ Private sector } \\
\hline Payments & 4.2 & 3.8 & 3.7 & 1.6 & 0.0 \\
\hline \multicolumn{6}{|l|}{ Memorandum item: } \\
\hline Total debt service due & 185.2 & 215.1 & 160.6 & 137.7 & 133.5 \\
\hline Actual debt service payments & 4.6 & 11.8 & 9.8 & 6.3 & 6.1 \\
\hline
\end{tabular}

Sources: Fund staff estimates based on information provided by the Sudanese authorities.

1/ Including GRA charges, Trust Fund interest, special charges, arrangement charges, and total SDR charges.

$2 /$ Includes estimates of late interest and interest on short-term credits.

3 / Including payments of arrears on repurchases and burden sharing refunds. 
Table 34. Sudan: Imports of Petroleum Products, and Landed Costs, 1994/95-97

\begin{tabular}{|c|c|c|c|c|}
\hline & $1994 / 95$ & 1995 & 1996 & 1997 \\
\hline \multicolumn{5}{|c|}{$\begin{array}{l}\text { Import values c.i.f. } \\
\text { (US\$ millions) }\end{array}$} \\
\hline Crude oil & 49 & 1.13 & 124 & 111 \\
\hline Benzine & 7 & 11 & 18 & 30 \\
\hline Superbenzine & 0 & 0 & 0 & 0 \\
\hline Gas Oil & 42 & 70 & 108 & 102 \\
\hline Kerosene & 0 & 0 & 0 & 0 \\
\hline Jet Al & 5 & 1 & 7 & 9 \\
\hline FF 1,500 & 0 & 0 & 0 & 0 \\
\hline FF 3,500 & 7 & 5 & 2 & 0 \\
\hline Diesel & 0 & 0 & 0 & 0 \\
\hline LPG & 2 & 4 & 6 & 9 \\
\hline Avgas & 1 & 0 & 0 & 0 \\
\hline \multicolumn{5}{|c|}{ Import volumes $(000 \mathrm{mt})$} \\
\hline Crude oil & 338 & 971 & 712 & 609 \\
\hline Benzine & 35 & 80 & 76 & 117 \\
\hline Superbenzine & 0 & 0 & 0 & 0 \\
\hline Gas Oil & 254 & 528 & 480 & 531 \\
\hline Kerosene & 0 & 2 & 0 & 0 \\
\hline Jet A1 & 25 & 7 & 25 & 9 \\
\hline FF 1,500 & 0 & 0 & 0 & $\mathbf{0}$ \\
\hline FF 3,500 & 79 & 58 & 27 & 0 \\
\hline Diesel & 0 & 0 & 0 & $\mathbf{0}$ \\
\hline LPG & 6 & 11 & 17 & 20 \\
\hline Avgas & 2 & 0 & 0 & 0 \\
\hline \multicolumn{5}{|c|}{$\begin{array}{l}\text { Domestic refining of } \\
\text { Imported crude (000 mt) }\end{array}$} \\
\hline Benzine & 54 & 114 & 137 & 89 \\
\hline Superbenzine & 0 & 0 & 0 & 0 \\
\hline Gas Oil & 98 & 214 & 210 & 157 \\
\hline Kerosene & 0 & 0 & 0 & 0 \\
\hline Jet Al & 24 & 40 & 41 & 43 \\
\hline FF 1,500 & 0 & 303 & 288 & 231 \\
\hline FF 3,500 & 145 & 2 & $\mathbf{0}$ & 0 \\
\hline Diesel & 0 & 8 & 9 & 4 \\
\hline LPG & 3 & 7 & 9 & 4 \\
\hline Avgas & $\mathbf{0}$ & 0 & 0 & 0 \\
\hline \multicolumn{5}{|c|}{ Landed cost (LSd per ton) 1/ } \\
\hline Crude oil & $\cdots$ & $\cdots$ & 281,648 & 298,100 \\
\hline Benzine & $\ldots$ & $\ldots$ & 378,252 & 458,732 \\
\hline Superbenzine & $\ldots$ & $\ldots$ & & \\
\hline Gas Oil & $\ldots$ & $\cdots$ & 362,935 & 430,646 \\
\hline Kerosene & $\ldots$ & $\ldots$ & 379,103 & \\
\hline Jet A1 & $\ldots$ & $\ldots$ & 379,103 & 338,043 \\
\hline FF 1,500 & $\ldots$ & $\ldots$ & & $\cdots$ \\
\hline FF 3,500 & $\ldots$ & $\ldots$ & 229,754 & $\ldots$ \\
\hline Diesel & $\ldots$ & $\ldots$ & & \\
\hline LPG & $\ldots$ & $\ldots$ & 658,630 & 725,128 \\
\hline Avgas & $\ldots$ & $\ldots$ & 854,035 & $\ldots$ \\
\hline
\end{tabular}

Source: General petroleum Corporation

1 Includes bank charges, operating and administrative expenses, quay dues, and interest charges. 
Table 35. Average Capacity Utilization in the Industrial Sector, 1993/94-97

(In percent)

\begin{tabular}{llllll}
\hline $1993 / 94$ & $1994 / 95$ & 1995 & 1996 & 1997 \\
\hline
\end{tabular}

Food and beverages

Flour 35 25 29

Sugar 62

63

69

68

74

Vegetable oils

15

15

12

17

19

Soft drinks

46

48

55

42

50

Textiles and apparel

Textiles

Shoes

25

7

10

8

9

10

30

34

28

31

Other consumer goods

Cigarettes

26

19

18

23

Intermediate goods

Cement

48

65

59

75

88

Source: Ministry of Industry. 


\section{The Tax System of Sudan}

This appendix describes the operation and performance of the main taxes levied in Sudan up to 1997. It also highlights some of the important factors that have affected the operation of certain specific taxes.

\section{Operation and performance of taxes}

\section{a. Personal income tax}

The personal income tax (PIT) is paid annually by residents on all income earned in Sudan or abroad, including wages, salaries, leave and sick pay, and all other remuneration for employment or services rendered. However, interest and domestic dividends, employers' contributions to retirement and medical schemes, and housing and transport allowances are excluded. Individual income taxes are "pay-as-you-earn" basis, and are deducted by employers who are obliged to submit collected monthly revenues by the 15 th day of the following month. In the case of a refund, employees may file their returns after December 31.

As of January 1997, the applicable personal income tax rates are as follows:

$\begin{array}{lll}\text { First } & \text { LSd } 240,000 & \text { exempted } \\ \text { next } & \text { LSd } 12,000 & 5 \text { percent } \\ " & \text { LSd } 15,000 & 10 \text { percent } \\ " & \text { LSd } 24,000 & 15 \text { percent } \\ " & \text { LSd } 36,000 & 20 \text { percent } \\ " & \text { LSd } 36,000 & 25 \text { percent } \\ \text { exceeding } & \text { LSd 363,000 } & 30 \text { percent }\end{array}$

Effective January 1997, the minimum threshold was raised from LSd 144,000 to LSd 240,000, while the highest income bracket was increased from to LSd 276,000 to LSd 363,000.

Overall, less than 300,000 individuals are subject to the PIT, but only 50,000 tax declarations are normally submitted. This is due to the fact that most employers withhold the tax directly from the wages and salaries, and also because other additional sources of income are usually insignificant. Between 1982/83 and 1997, the revenue from the personal income tax as a share of GDP has declined almost continuously (from 0.6 percent to 0.1 percent).

\section{b. Business profit tox}

The business profit tax, which was based on the Income Tax Act of 1986, is levied on net business profits of individual entrepreneurs, limited public and private companies, partnerships, trusts and associations, banks, and insurance companies. Profit taxes are 
calculated on the basis of profits earned over the year ended December 31 and companies must file their returns by March 31. The first installment is due by April 30, and the second by October 31 .

The schedule of rates is currently as follows:

\section{(1) Individual entrepreneurs}

a. Non-agriculture

$\begin{array}{crc}\text { First } & \text { LSd } 240,000 & \text { exempted } \\ \text { next } & \text { LSd } 150,000 & 10 \text { percent } \\ " & \text { LSd } 250,000 & 20 \text { percent } \\ " & \text { LSd } 500,000 & 30 \text { percent } \\ \text { exceeding } & \text { LSd } 1,140,000 & 40 \text { percent }\end{array}$

b. Agriculture (2 percent on gross income)

The Taxation Bureau of the Ministry of Finance and Economic Planning (TB) estimates that about 170,000 individual entrepreneurs are currently subject to this tax. Owing to the high rate of inflation and the lack of corresponding adjustments in the nominal tax brackets, about 95 percent of taxpayers currently fall in the highest bracket. Hence, the profit tax has become regressive, posing a relatively higher burden on smaller or medium level business incomes. In January 1997, the minimum threshold was raised from LSd 100,000 to LSd 240,000. Revenue from this tax has been transferred in 1997 to the state governments.

\section{(2) Private companies, including banks and insurance companies}

Since July 1995 , the net income of private companies is taxed at 40 percent while that of banks and insurance companies are taxed at 45 percent. The TB estimates that there are about 4,500 registered enterprises which are subject to this tax.

Revenue from the profit tax fell during the 1980s primarily because of two factors. First, the existence of generous exemptions and immunities led to a decline of revenue. A broad range of concessions and exemptions were granted in accordance with the 1989' Taxation Act and the 1991 Investment Encouragement Act. Over 60 companies and several banks currently benefit from these tax exemptions. ${ }^{1}$ The loss in revenues from these specific

${ }^{1}$ For example, companies operating under the 1991 Investment Encouragement Act are granted a five to ten years tax holiday. This Act subjects tax concessions to the fulfillment of any of several conditions, among which are: (a) the contribution of the investment project to the increase of national income; (b) the removal of "any bottlenecks obstructing 
exemptions and immunities was large. Furthermore exemptions were extended also to cooperatives dealing with trade, transport, agriculture, and other activities, and 53 charity organizations which undertook commercial activities. In March 1995, exemptions were eliminated for cooperatives and charities that operate on commercial basis and in July 1995, a 1 percent development tax on the income of enterprises benefiting from tax holiday was introduced (strategic projects are exempted from this tax). In 1997, actual collection was LSd 2.9 billion. Second; the decline in revenues was also caused by the large lags in tax payments. To minimize the loss from such lags, in March 1995, a ministerial decree was issued stating that business profit tax and rental tax payments would be made at the time of tax payer declaration or presentation of audited accounts. The main factors that still exist behind these delays are the weak penalty system and the widespread utilization of the appeal system.

\section{c. Tax on Sudanese working abroad}

The current annual rates of taxation are as follows:

\section{Rates as of December 1997}

(In U.S. dollars)

Laborers

Employees

Professionals

University educators

Businessmen
150

350

$500-800$

$500-800$

2,000

The rates are enforced by requiring that citizens obtain an exit visa verifying tax payment before leaving Sudan and that passports be renewed every two years. About 200,000 expatriates have complied with this tax in recent years.

\section{d. Agricultural tox}

Farmers are subject to local taxation (by regional and provincial governments). Most of these taxes are collected during the harvest period from November to April.

\section{${ }^{1}$ (...continued)}

development;" (c) the "consolidation" of economic developments; and (d) and increase in employment. After an initial effective period of five years, the tax concession can be extended for additional five years in the case of large investments. Furthermore, the Act also exempts these investment schemes from customs duties and excises on equipments, machineries, and spare parts. Losses may be carried forward and deducted from profits for a period of five years. 
In July 1993, the agricultural tax rate collected by the Central Government was lowered from 5 percent to 2 percent, and beginning in 1996, the amount collected by the Central Government was transferred to the state governments.

\section{e. Capital gains tax}

In July 1995, the capital gains tax rate was lowered from 10 percent to 5 percent for real estate and from 5 percent to 2.5 percent for cars, and is levied on the difference between the sales and purchase price without adjustment for inflation. Revenue from this tax has been transferred in 1997 to the state governments.

\section{f. Sales tax}

Most items manufactured in Sudan are subject to a sales tax of 10 percent, with the exception of medicine, food, and vegetable oils. Both the sales and excise taxes are collected at the factory by the Customs department. Since 1996, the amount collected from sales and excise taxes on a number of commodities have been transferred to the state governments. Inspectors located within the factory issue forms certifying that taxes have been paid before allowing goods to be shipped. This tax is not applicable to corresponding imports, which are subject to customs duty and consumption tax. The rates for the major items are listed in Appendix I, Table 7.

\section{(1) Sales tax on services}

The tax on services is charged at a rate of 20 percent for hotels, restaurants, and club services. An airport departure tax is levied on all international flights by the Civil Aviation Authority. In October 1994, this tax was raised to LSd 5,000 per passenger and to US $\$ 10.00$ per non-resident. In October 1996, the airport departure was raised to US\$20.00 per non-resident.

\section{(2) Excise tax}

The excise tax is also levied on several domestically manufactured products at a variety of ad valorem rates ranging from 2 percent to 150 percent. As mentioned above, beginning 1996, the revenue generated from excise tax on a number of commodities is transferred to the state governments. Currently, there are 7 different excise rates applicable to about 20 goods.

\section{g. Taxes on international trade}

Sudan has relied considerably on taxes on international trade as a source of income; these include import and export duties, and the consumption tax. Their contribution to total tax collection increased from 37 percent in 1995 to 51 percent in 1997. This increase was due 
to the merging (in April 1997) of the customs valuation rate with the official exchange rate and the increase in imports.

\section{(1) Import duties}

All import duties are ad valorem and are applied to a fair market c.i.f import price. The import duty rates range from 5 percent to 250 percent (Table 8 ). The revenue generated from import duties are transferred to the Central Government.

\section{(2) Export duties}

All export duty rates are ad valorem and are calculated on free along side (f.a.s) value. Until end-1997, with the exception of cotton and gum arabic, export duties were applied according to two bands 0-3 percent or 5 percent. Export duties on cotton and gum arabic were charged at the rate of 10 percent. Beginning in January 1998, export duty rates were reduced by 3 percentage points.

\section{(3) Consumption tax}

The consumption tax rate is levied at a rate of 10 percent on a broad range of items. Items exempted from the consumption tax include the following: wheat flour, printed books and booklets, sacks and bags for packing, and medical supplies (Table 8).

\section{(4) Defense tax}

Beginning in August 1997, a defense tax at a rate of 4 percent was reinstated, on a temporary basis, on a number of commodities equivalent to about 40 percent of total imports. Revenue from the defense tax accounted for about 0.2 percent of GDP in 1997.

\section{(5) Services tax}

Instituted in 1994 as a levy of 5 percent of c.i.f. import value on products previously subject to quantitative restrictions.

\section{h. Other toxes}

Other taxes include various levies and fees, such as stamp duties and car license fees. Their contribution to the budget has been insignificant. In Appendix I Table 16, these revenues are combined with those from the agriculture tax, and have yielded no more than 0.5 percent of GDP since the mid-1980s. The stamp duty is charged at various rates on more than 260 instruments of various kinds. 